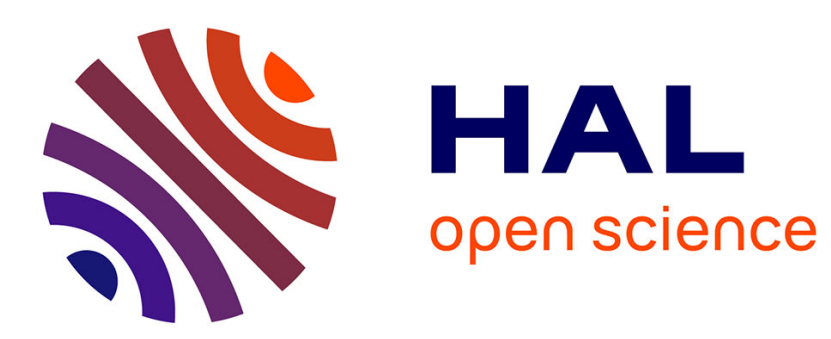

\title{
Uniqueness of multiplicative determinants on elliptic pseudodifferential operators
}

\author{
Jean-Marie Lescure, Sylvie Paycha
}

\section{To cite this version:}

Jean-Marie Lescure, Sylvie Paycha. Uniqueness of multiplicative determinants on elliptic pseudodifferential operators. Proc. of the London Math. Soc., 2007, 94, pp.772-812. hal-00483875

\section{HAL Id: hal-00483875 \\ https://hal.science/hal-00483875}

Submitted on 23 Nov 2019

HAL is a multi-disciplinary open access archive for the deposit and dissemination of scientific research documents, whether they are published or not. The documents may come from teaching and research institutions in France or abroad, or from public or private research centers.
L'archive ouverte pluridisciplinaire HAL, est destinée au dépôt et à la diffusion de documents scientifiques de niveau recherche, publiés ou non, émanant des établissements d'enseignement et de recherche français ou étrangers, des laboratoires publics ou privés. 


\title{
Uniqueness of multiplicative determinants on elliptic pseudodifferential operators
}

\author{
Jean-Marie LESCURE, Sylvie PAYCHA
}

September 21, 2006

\begin{abstract}
We describe all multiplicative determinants on the pathwise connected component of identity in the group of invertible classical pseudodifferential operators on a closed manifold, that are continuous along continuous paths and the restriction to zero order operators of which is of class $C^{1}$. This boils down to a description of all traces on zero order classical pseudodifferential operators, which turn out to be linear combinations of the Wodzicki residue [W] and leading symbol traces introduced in [PR1], both of which are continuous. Consequently, multiplicative determinants are parametrized by the residue determinant [W87, Sc] and a new "leading symbol determinant", both of which are expressed in terms of a homogeneous component of the symbol of the logarithm of the operator.
\end{abstract}

\section{Introduction}

There are at least two known determinants on elliptic classical pseudodifferential operators (with spectral cut) on a closed $n$-dimensional manifold $M$,

- the $\zeta$-determinant

$$
\operatorname{det}_{\zeta}(A)=e^{-\zeta_{A}^{\prime}(0)}
$$

where $z \mapsto \zeta_{A}(z)$ is the $\zeta$-function of $A$, an extension of the ordinary determinant introduced by Ray and Singer [RS] on the mathematical side and first used by Hawkings [Ha] on the physics side in order to make sense of partition functions,

- the residue determinant $[\mathrm{Sc}]$

$$
\operatorname{det}_{\text {res }}(A)=e^{\widetilde{\operatorname{res}}(\log A)}
$$

which coincides on order zero operators with the multiplicative residue or exotic (log-) determinant introduced by Wodzicki [W87] (see also [Ka] for a review). Here $\widetilde{\text { res }}(\log A)$ is the extended Wodzicki residue $[\mathrm{O}]$

$$
\widetilde{\operatorname{res}}(\log A)=\frac{1}{2 \pi^{n}} \int_{S^{*} M} \operatorname{tr}_{x}\left(\sigma_{-n}(\log A)(x, \xi)\right) d_{S} \xi
$$


of the $\log$ arithm $\log A=\left.\frac{\partial}{\partial z}\right|_{z=0} A^{z}$ where $A^{z}$ is the complex power in the sense of Seeley. $d_{S} \xi$ stands for the volume measure on the cotangent unit sphere $S^{*} M$ induced by the canonical volume measure on the cotangent bundle $T^{*} M$ and $\sigma_{j}(A)(x, \xi) \in C^{\infty}\left(T^{*} M\right)$ for the (positively) homogeneous part of degree $j$ of the symbol $\sigma(A)$ of $A \in C \ell(M, E)$.

Whereas the residue determinant is multiplicative as a result of the invariance of the extended Wodzicki residue under the adjoint action of the group of invertible classical pseudodifferential operators, the $\zeta$-determinant presents a multiplicative anomaly investigated in [KV1, KV2], [O].

The present article aims at a full description of multiplicative determinants

$$
\text { Det }: \tilde{C} \ell^{*}(M, E) \rightarrow \mathbb{C}^{*},
$$

defined on the pathwise connected component to identity $\tilde{C} \ell^{*}(M, E)$ of the group $C \ell^{*}(M, E)$ of invertible classical pseudodifferential operators acting on smooth sections of a vector bundle $E$ over an $n$-dimensional closed manifold $M$, with the additional regularity requirement that Det restricts to a $C^{1}$-functional on zero order operators and that it is continuous on continuous paths of classical pseudodifferential operators of any order.

The restriction $\operatorname{Det}^{0}$ of such a multiplicative determinant Det to operators of zero order defines a character on the pathwise connected component to identity $\tilde{C} \ell^{0, *}(M, E)$ of the Fréchet Lie group $C \ell^{0, *}(M, E)$ of invertible zero order elliptic operators. Det being of class $C^{1}$, so is $\operatorname{Det}^{0}$ and the differential at the identity of the latter induces a continuous trace

$$
\operatorname{Tr}:=D_{1} \operatorname{Det}^{0}: C \ell^{0}(M, E) \rightarrow \mathbb{C}
$$

on the Lie algebra $C \ell^{0}(M, E)$ of $C \ell^{0, *}(M, E)$ which consists of zero order classical pseudodifferential operators acting on smooth sections of $E$. The problem therefore boils down to looking for continuous traces on $C \ell^{0}(M, E)$.

Adapting methods developed by Wodzicki [W87] to compute the homology of differential operators and by Brylinski and Getzler in [BG] to compute the homology of the algebra $C \ell^{\mathbb{Z}}(M, E)$ of classical pseudodifferential operators acting on smooth sections $E$ with integer order, we compute the zero-th homology of the algebra $C \ell^{0}(M, E)$ of zero-th order classical pseudodifferential operators and deduce from there (see Theorem 4) a uniqueness result for traces on $C \ell^{0}(M, E)$.

Theorem 1 Traces on $C \ell^{0}(M, E)$ are linear combinations of the Wodzicki residue and of the leading symbol traces ${ }^{1}$ :

$$
\operatorname{Tr}_{0}^{\tau}(A)=\tau\left(\operatorname{tr}_{x}\left(\sigma_{0}(A)(x, \xi)\right)\right)
$$

where $\tau \in C^{\infty}\left(S^{*} M\right)^{\prime}$ is a current on the cotangent unit sphere $S^{*} M$. They are continuous for the Fréchet topology on $C \ell^{0}(M, E)$.

The proof uses the spectral sequence introduced by Wodzicki, Brylinski and Getzler [W87, BG] to prove the uniqueness of traces on the whole algebra

\footnotetext{
${ }^{1}$ which corresponds to the restriction to $C \ell^{0}(M, E)$ of the unique (up to a multiplicative factor and for $\operatorname{dim} M>1$ ) continuous trace on the full algebra $C \ell(M, E)$
} 
$C \ell^{\mathbb{Z}}(M, E)$. Nevertheless, the constraint on the order of the operators considered here gives rise to a different behavior of the spectral sequence: for instance, there are new non vanishing tems at the $E_{2}$-step which prevent us from drawing direct conclusions concerning degeneracy as in [W87, BG].

On the basis of general results on determinant maps on Lie groups associated with traces on their Lie algebras- which we need to extend to an infinite dimensional setting including Fréchet Lie groups (see Theorem 3)- we show (see Theorem 5) a uniqueness result for multiplicative determinants of class $C^{1}$ on $\tilde{C} \ell^{0, *}(M, E):=\tilde{C} \ell^{*}(M, E) \cap C \ell^{0}$. They are of the form

$$
\operatorname{det}(A)=e^{\alpha \int_{0}^{1} \operatorname{res}\left(\gamma_{A}^{-1}(t) \dot{\gamma}_{A}(t)\right) d t+\beta \int_{0}^{1} \operatorname{Tr}_{0}^{\tau}\left(\gamma_{A}^{-1}(t) \dot{\gamma}_{A}(t)\right) d t}, \quad \alpha, \beta \in \mathbb{R},
$$

where $\gamma_{A}$ is a differentiable path such that $\gamma(0)=I d, \gamma(1)=A$. When $\beta=0$ this yields back the multiplicative residue introduced by Wodzicki. When $\alpha=0$ this yields a new type of determinant which we refer to as leading symbol determinants.

Extending these multiplicative determinants from zero order to positive order classical pseudodifferential operators requires extending the Wodzicki residue and the leading symbol traces to logarithmic operator in the sense of $[\mathrm{O}]$. Logarithmic operators of type $q$ differ from zero order classical pseudodifferential operators by the $\operatorname{logarithm} \log Q=\left.\frac{d}{d z}\right|_{z=0} Q^{z}$ of some elliptic operator $Q \in C \ell^{*}(M, E)$ of order $q$ with spectral cut. There is a natural extension of differentiability of paths in $C \ell^{0}(M, E)$ for the Fréchet topology to differentiability of paths in $C \ell(M, E)$ with (varying) differentiable order and such a path $\gamma \in C \ell^{*}(M, E)$ has logarithmic differential $\gamma^{-1} \dot{\gamma}$ given by a logarithmic operator.

Using a natural extension $\widetilde{\text { res }}$ of the Wodzicki residue res to logarithmic operators [O], it makes sense to define the residue determinant of $A$ for $A \in \tilde{C} \ell^{*}(M, E)$ of any order by:

$$
\operatorname{det}_{\text {res }}(A)=e^{\int_{0}^{1} \widetilde{\operatorname{es}}\left(\gamma_{A}^{-1}(t) \dot{\gamma}_{A}(t)\right) d t}
$$

with $\gamma_{A}$ a differentiable path in $C \ell^{*}(M, E)$ linking 1 to $A$.

We show that leading symbol traces $\operatorname{Tr}_{0}^{\tau}$ also extend to linear forms $\widetilde{\operatorname{Tr}_{0}^{\tau}}$ on logarithmic operators so that we can define the leading symbol determinant of an operator $A \in \tilde{C} \ell^{*}(M, E)$ of any order by:

$$
\operatorname{det}_{0}^{\tau}(A)=e^{\int_{0}^{1} \widetilde{\operatorname{Tr}_{0}^{\tau}}\left(\gamma_{A}^{-1}(t) \dot{\gamma}_{A}(t)\right) d t} .
$$

Both $\operatorname{det}_{\text {res }}$ and $\operatorname{det}_{0}^{\tau}$ are independent of the choice of path $\gamma_{A}$ linking 1 to $A$. These extended determinants remain multiplicative as a result of the Adinvariance under the adjoint action of the group of invertible classical pseudodifferential operators of the extended Wodzicki residue and leading symbol traces. The uniqueness result on continuous linear traces on zero order operators implies a uniqueness result on these extended determinants (see Theorem 7).

Theorem 2 Multiplicative maps $\Lambda: \tilde{C}^{*}(M, E) \rightarrow \mathbb{C}^{*}$ that are continuous along continuous paths in $\tilde{C} \ell^{*}(M, E)$ and the restriction to $\tilde{C} \ell^{0, *}(M, E)$ of which 
is of class $C^{1}$ in the Fréchet topology, read:

$$
\Lambda(A)=K^{a} e^{\alpha \int_{0}^{1} \widetilde{\operatorname{res}}\left(\gamma_{A}^{-1}(t) \dot{\gamma}_{A}(t)\right) d t+\beta \int_{0}^{1} \widetilde{\operatorname{Tr}_{0}^{\tau}}\left(\gamma_{A}^{-1}(t) \dot{\gamma}_{A}(t)\right) d t}, \quad \alpha, \beta, K \in \mathbb{C}
$$

where $A$ is an operator of order $a$ and $\gamma_{A}$ a differentiable path in $C \ell^{*}(M, E)$ linking 1 to $A$.

An invertible operator $A$ with spectral cut $\theta$ lies in $\tilde{C} \ell^{*}(M, E)$ since there is a path $\gamma_{A, \theta}(t)=A_{\theta}^{t}, t \in[0,1]$ linking 1 to $A$. Using the invariance of $\widetilde{\text { res }}$ and $\widetilde{\operatorname{Tr}}_{0}^{\tau}$ under the adjoint action of the group of invertible classical pseudodifferential operators, we show that the residue determinant coincides with the one investigated by Scott [Sc]

$$
\operatorname{det}_{\text {res }}(A)=e^{\widetilde{\operatorname{res}}(\log A)}
$$

and that leading symbol determinant read

$$
\operatorname{det}_{0}^{\tau}(A)=e^{\widetilde{\operatorname{tr}}_{0}^{\tau}(\log A)} .
$$

These definitions are independent of the choice of spectral cut $\theta$ since they are independent of the choice of path $\gamma_{A, \theta}(t)=A_{\theta}^{t}, t \in[0,1]$.

Let us comment on the relevance of these multiplicative determinants. The $\zeta$-determinant is used in physics to provide an Ansatz for partition functions on the basis of a formula of the type:

$$
\int_{\mathcal{C}} e^{-\frac{1}{2}\langle A \phi, \phi\rangle} \mathcal{D} \phi:=\operatorname{det}_{\zeta}(A)^{-\frac{1}{2}}
$$

where $\phi$ is a configuration seen here as a section of a vector bundle $E, \mathcal{D} \phi$ a heuristic "path space measure" on the configuration space $\mathcal{C}$, and $A$ an admissible elliptic operator acting on sections of $E$.

One could replace the $\zeta$-determinant in the above Ansatz by one of these multiplicative determinants, i.e. a residue or a leading symbol determinant. Doing so would bear some advantages:

- this would lead to local formulae since both the Wodzicki residue and the leading symbol trace from which these determinants are built are local, a feature which is not usually expected when using the $\zeta$-determinant which is non local,

- it would in certain cases avoid the ocurrence of anomalies which often arise when using the $\zeta$-determinant.

For example, in the usual $\zeta$-determinant setup, conformal anomalies [PR2] arise from a $\zeta$-regularized trace of the multiplication operator by a smooth function $f$ as a result of the conformal transformation $g \mapsto e^{2 f} g$ where $g$ is a Riemannian metric on the underlying closed manifold $M$. Were we to replace the $\zeta$-determinant by the residue determinant, the underlying trace would be a Wodzicki residue which vanishes on multiplication operators. Were we to choose instead a leading symbol determinant, the conformal anomaly would be proportional to the mean value of $f$ w.r. to the volume measure on $M$. 
However convenient these mutiplicative determinants may be, they do not seem to capture new interesting geometric properties or lead to new interesting physical quantities. One one hand, the leading symbol determinant is too coarse to distinguish between two admissible elliptic operators of same order; e.g. it does not distinguish between the Bochner operator $d d^{*}+d^{*} d$ and the generalized Laplace operator $\nabla^{*} \nabla$ on 1-forms. On the other hand, as one could expect, the Wodzicki residue determinant seems to recapture known anomalies which arise from using the $\zeta$-determinant as an Ansatz for the partition function. Indeed, as was pointed out in $[\mathrm{Sc}]$, on an admissible differential operator the logarithm of the residue determinant coincides (up to a sign) with the value of the $\zeta$-function at 0 of the operator. In a supersymmetric setup, the residue determinant therefore typically captures the index of a Dirac type operator which usually detects chiral anomalies.

The complete description of multiplicative determinants presented in this paper therefore somehow settles the issue of why not choose a multiplicative determinant instead of the $\zeta$-determinant as an Ansatz for partition functions.

The paper is organized as follows:

1. Traces and associated determinants

- Lie groups with an exponential mapping

- From a trace on the Lie algebra to a determinant on the Lie group and vice versa

2. Traces on zero order classical pseudodifferential operators

- A Lie group structure on zero order invertible classical pseudodifferential operators

- Hochschild homology of the algebra of zero order classical pseudodifferential operators

- A uniqueness result for traces on zero order classical pseudodifferential operators

3. Determinants on zero order invertible pseudodifferential operators

- The fundamental group of the class of zero order invertible pseudodifferential operators

- A uniqueness result for multiplicative determinants on zero order operators

4. Multiplicative determinants on invertible classical pseudodifferential operators of all orders

- Logarithmic operators

- Traces on $C \ell^{0}(M, E)$ extended to logarithmic operators

- Continuous paths of varying order

- A uniqueness result for multiplicative determinants on operators of all orders 


\section{Acknowledgements}

The second author would like to thank Simon Scott for his useful comments on a preliminary version of this article and Mikhail Shubin for interesting discussions around traces on algebras of pseudodifferential operators.

\section{Traces and associated determinants}

This section extends known results on the construction of determinants associated with traces from the case of Banach Lie groups investigated in [dLHS] to any infinite dimensional Lie groups with an exponential mapping.

\subsection{Lie groups with an exponential mapping}

In this paragraph, we closely follow the presentation of $[\mathrm{KM}]$ concerning infinite dimensional Lie groups. Let us first introduce some notations.

Let $\mathcal{G}$ be a Lie group, i.e. a (possibly infinite dimensional) smooth manifold equipped with a group structure such that the multiplication $(g, h) \mapsto g h$ and the inversion $g \mapsto g^{-1}$ are smooth. ${ }^{2}$ Let

$$
\begin{aligned}
L_{g}: \mathcal{G} & \rightarrow \mathcal{G} \\
h & \mapsto g h
\end{aligned}
$$

and

$$
\begin{aligned}
R_{g}: \mathcal{G} & \rightarrow \mathcal{G} \\
h & \mapsto h g
\end{aligned}
$$

denote the corresponding left and right multiplication respectively. As usual, we identify the algebra of left invariant fields $\Xi_{L}(\mathcal{G}):=\left\{\xi \in C^{\infty}(T \mathcal{G}),\left(L_{g}\right)_{*} \xi=\right.$ $\xi \forall g \in \mathcal{G}\}$ with the Lie algebra of $\mathcal{G}$ which we denote by Lie $(\mathcal{G})$. With this identification, the bracket on Lie $(\mathcal{G})$ can be written in terms of the operator bracket and Lie derivative

$$
[\xi, \eta]=\left[\mathcal{L}_{\xi}, \mathcal{L}_{\eta}\right] \quad \forall \xi, \eta \in \operatorname{Lie}(\mathcal{G}) .
$$

Lemma 1 Let $\mathcal{G}$ and $\mathcal{H}$ be two Lie groups.

- $A \operatorname{map} \Phi: U \subset \mathcal{G} \rightarrow \mathcal{H}$ defined on a neighborhood $U$ of e, which is differentiable at $e_{\mathcal{G}}$ and multiplicative (and hence $\Phi\left(e_{\mathcal{G}}\right)=e_{\mathcal{H}}$ ) i.e.

$$
\Phi(g h)=\Phi(g) \Phi(h) \quad \forall(g, h) \in U^{2} \quad \text { such that } g h \in U,
$$

induces a Lie algebra homomorphism $\phi: \operatorname{Lie}(\mathcal{G}) \rightarrow \operatorname{Lie}(\mathcal{H})$ with

$$
\phi=D_{e} \Phi .
$$

$\phi$ is continuous whenever $\Phi$ is of class $C^{1}$.

\footnotetext{
${ }^{2}$ If an implicit function theorem is available, then smoothness of the inversion follows from smoothness of the mutliplication.
} 
- In particular, a smooth homomorphism $\Phi: \mathcal{G} \rightarrow \mathcal{H}$ of Lie groups induces a cotninuous Lie algebra homomorphism $\phi: \operatorname{Lie}(\mathcal{G}) \rightarrow \operatorname{Lie}(\mathcal{H})$ with

$$
\phi=D_{e} \Phi
$$

Proof: Since $\Phi$ is multiplicative we have

$$
\Phi \circ L_{g}=L_{\Phi(g)} \circ \Phi
$$

and hence for any $\xi \in \operatorname{Lie}(\mathcal{G})$ and $g \in \mathcal{G}$ we have

$$
\begin{aligned}
\mathcal{L}_{\phi(\xi)}(\Phi(g)) & =D_{e}\left(L_{\Phi(g)}\right) D_{e} \Phi(\xi) \\
& =D_{e}\left(L_{\Phi(g)} \circ \Phi\right)(\xi) \\
& =D_{e}\left(\Phi \circ L_{g}\right)(\xi) \\
& =D_{g} \Phi\left(\left(D_{e} L_{g}\right)(\xi)\right) \\
& =D_{g} \Phi \mathcal{L}_{\xi}(g) .
\end{aligned}
$$

Thus, for any $\xi \in \operatorname{Lie}(\mathcal{G})$

$$
\mathcal{L}_{\phi(\xi)} \circ \Phi=D \Phi \circ \mathcal{L}_{\xi},
$$

from which it follows that for any $\xi, \eta \in \operatorname{Lie}(\mathcal{G})$

$$
\begin{aligned}
\mathcal{L}_{[\phi(\xi), \phi(\eta)]} \circ \Phi & =\left[\mathcal{L}_{\phi(\xi)}, \mathcal{L}_{\phi(\eta)}\right] \circ \Phi \\
& =\left[D \Phi \circ \mathcal{L}_{\xi}, D \Phi \circ \mathcal{L}_{\eta}\right] \\
& =D \Phi \circ\left[\mathcal{L}_{\xi}, \mathcal{L}_{\eta}\right] \\
& =\phi([\xi, \eta]) .
\end{aligned}
$$

This shows that $\phi$ preserves the bracket and hence that it is a Lie algebra homomorphism.

Following $[\mathrm{KM}]$ we set

Definition 1 A Lie group $\mathcal{G}$ admits an exponential mapping if there exists a smooth mapping

$$
\operatorname{Exp}: \operatorname{Lie}(\mathcal{G}) \rightarrow \mathcal{G}
$$

such that $t \mapsto \operatorname{Exp}(t X)$ is a one-parameter subgroup with tangent vector $X$. $\operatorname{Exp}(0)=e_{\mathcal{G}}$ and $\operatorname{Exp}$ induces the identity map $D_{e} \operatorname{Exp}=I d_{\operatorname{Lie}(\mathcal{G})}$ on the corresponding Lie algebra.

Let $\Phi: \mathcal{G} \rightarrow \mathcal{H}$ be a smooth homomorphism between Lie groups admitting exponential mappings $\operatorname{Exp}_{\mathcal{G}}$ and $\operatorname{Exp}_{\mathcal{H}}$ respectively then the diagram

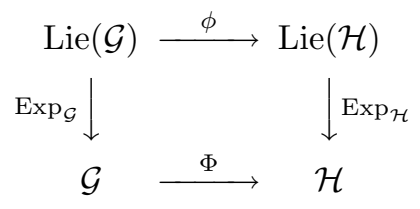

commutes. 
Remark 1 - If $E$ is a Banach space, then the Banach Lie group of all bounded automorphisms of $E$ is equipped with an exponential mapping given by the series $\operatorname{Exp}(X)=\sum_{i=0}^{\infty} \frac{X^{i}}{i !}$.

- All known smooth Fréchet Lie groups admit an exponential mapping although it is not known, according to $[K M]$, whether any smooth Fréchet Lie group does admit an exponential mapping.

- If $\mathcal{G}$ has an exponential mapping and if a suitable inverse function theorem is applicable, then Exp yields a diffeomorphism from a neighborhood of $0 \in \operatorname{Lie}(\mathcal{G})$ to a neighborhood of $e \in \mathcal{G}$. This holds for smooth Banach Lie groups and for gauge groups but is wrong for diffeomorphism groups (see e.g. $[K M])$.

\subsection{From a trace on the Lie algebra to a determinant on the Lie group and vice versa}

For any $g \in \mathcal{G}$

$$
\operatorname{Int}_{g}:=L_{g^{-1}} \circ R_{g}=R_{g} \circ L_{g^{-1}} \in \operatorname{Aut}(\mathcal{G})
$$

induces a map

$$
\operatorname{Ad}_{g}:=D_{e} \operatorname{Int}_{g}=D g L_{g^{-1}} \circ D_{e} R_{g} \in \operatorname{Aut}(\operatorname{Lie}(\mathcal{G}))
$$

which in turn gives rise to a map

$$
\operatorname{ad}_{v}:=\left.\frac{d}{d t}\right|_{t=0} \operatorname{Ad}_{\gamma_{v}(t)} \in \operatorname{Hom}(\operatorname{Lie}(\mathcal{G}))
$$

where $\gamma_{v}(t)$ is any one-parameter semi-group generated by $v$. Clearly,

$$
[u, v]=a d_{v}(u) \quad \forall u, v \in \operatorname{Lie}(\mathcal{G}) .
$$

Definition $2 \quad$ - A (continuous) trace on a (topological) Lie algebra $(\mathcal{A},[\cdot, \cdot])$ (i.e. the bracket is a continuous bilinear map) is a (continuous) linear morphism $\lambda: \mathcal{A} \rightarrow \mathbb{C}$ of Lie algebras i.e. a (continuous) linear map which vanishes on brackets:

$$
\lambda([a, b])=0 \quad \forall a, b \in \mathcal{A}
$$

or equivalently whenever

$$
a d_{u}^{*} \lambda:=\lambda \circ a d_{u}=0 \quad \forall u \in \mathcal{A} .
$$

- Let $\mathcal{A}=\operatorname{Lie}(\mathcal{G})$ be the Lie algebra of a Lie group $\mathcal{G}$. $A$ map $\lambda: \mathcal{A} \rightarrow \mathbb{C}$ is said to be Ad-invariant whenever

$$
\operatorname{Ad}_{g}^{*} \lambda:=\lambda \circ \operatorname{Ad}_{g}=\lambda \quad \forall g \in \mathcal{G}
$$

- Let $\mathcal{G}$ be a Lie group and $\tilde{\mathcal{G}}$ its subgroup of elements pathwise connected to the identity $e_{\mathcal{G}}$. A determinant map on $\mathcal{G}$ is a group morphism $\Lambda: \tilde{\mathcal{G}} \rightarrow \mathbb{C}^{*}:$

$$
\forall g, h \in \tilde{\mathcal{G}} \quad \Lambda(g \cdot h)=\Lambda(g) \cdot \Lambda(h) .
$$


Remark 2 - If a determinant map is differentiable at $e_{\mathcal{G}}$ it is differentiable at every point $g \in \tilde{\mathcal{G}}$ since for every $h \in T_{e} \mathcal{G}$ we have

$$
\begin{aligned}
D_{g} \Lambda(h) & =D_{e} \Lambda(g h) \\
& =\Lambda(g) D_{e} \Lambda(h) .
\end{aligned}
$$

If moreover $\Lambda$ is continuous then so is the map $g \mapsto D_{g} \Lambda=\Lambda(g) D_{e} \Lambda$ and $\Lambda$ is of class $C^{1}$.

- The subgroup $\tilde{\mathcal{G}}$ is normal in $\mathcal{G}$ : in other words, it is invariant under $\operatorname{Ad}_{g}$ for any $g \in \mathcal{G}$.

If $\mathcal{A}$ equipped with the product $\cdot$ is an algebra with bracket $[a, b]=a \cdot b-b \cdot a$ and if $\mathcal{G} \subset \mathcal{A}$ then an ad-invariant map is also Ad-invariant. The following lemma yields the converse when $\mathcal{G}$ is a Lie group with exponential mapping.

Lemma $2 A$ continuous Ad-invariant map $\lambda:$ Lie $(\mathcal{G}) \rightarrow \mathbb{C}$ on the Lie algebra of a Lie group $\mathcal{G}$ with exponential mapping Exp yields a continuous ad-invariant map i.e. a continuous trace on the Lie algebra.

Let $\mathcal{A}$ be a topological algebra with bracket $[a, b]=a \cdot b-b \cdot a$ and let $\mathcal{G} \subset \mathcal{A}$ be $a$ Lie group with Lie algebra $(\mathcal{A},[\cdot, \cdot])$ and with exponential mapping. Then there is a one to one correspondance between continuous Ad-invariant map $\lambda: \mathcal{A} \rightarrow \mathbb{C}$ and continuous traces on $\mathcal{A}$.

Proof: We prove the first part of the lemma, since the second part then immediately follows. $\gamma_{v}(t)=\operatorname{Exp}(t v)$ is a one-parameter semi-group generated by $v$ and

$$
\begin{aligned}
\lambda([u, v]) & =\lambda\left(\operatorname{ad}_{v}(u)\right) \\
& =\lambda\left(\left.\frac{d}{d t}\right|_{t=0} \operatorname{Ad}_{\gamma_{v}(t)}(u)\right) \\
& =\lambda\left(\lim _{t \rightarrow 0} \frac{\operatorname{Ad}_{\gamma_{v}(t)}(u)-u}{t}\right) \\
& =\lim _{t \rightarrow 0} \lambda\left(\frac{\operatorname{Ad}_{\gamma_{v}(t)}(u)-u}{t}\right) \\
& =\left.\frac{d}{d t}\right|_{t=0} \lambda\left(\operatorname{Ad}_{\gamma_{v}(t)}(u)\right) \\
& =\left.\frac{d}{d t}\right|_{t=0} \lambda(u) \\
& =0,
\end{aligned}
$$

which ends the proof.

Setting $\mathcal{H}=\mathbb{C}^{*}$ and $\operatorname{Lie}(\mathcal{H})=\mathbb{C}$ in Lemma 1 provides a way to associate a trace with a determinant on a Lie group with an exponential mapping.

Proposition 1 Let $\mathcal{G}$ be a Lie group with an exponential map Exp. A determinant map $\Lambda$ on $\mathcal{G}$ (i.e. a multiplicative map $\Lambda: \tilde{\mathcal{G}} \rightarrow \mathbb{C}^{*}$ ) which is differentiable 
at $e \in \mathcal{G})$ (resp. of class $C^{1}$ on $\mathcal{G}$ ) induces a (resp. continuous) Ad-invariant map (and hence a trace)

$$
\lambda:=D_{e} \Lambda
$$

on its Lie algebra Lie $(\mathcal{G})$.

Proof: Since $\tilde{\mathcal{G}}$ is invariant under $A d_{g}$ for any $g \in \mathcal{G}$, for $u \in \operatorname{Lie}(\mathcal{G})$, the determinant map $\Lambda$ is well defined on $g^{-1} \operatorname{Exp}(t u) g$ for any $t \in \mathbb{R}$ and we have

$$
\begin{aligned}
\operatorname{Ad}_{g}^{*} D_{e} \Lambda(u) & =\left.\frac{d}{d t}\right|_{t=0} \operatorname{Ad}_{g}^{*} \Lambda(\operatorname{Exp}(t u)) \\
& =\left.\frac{d}{d t}\right|_{t=0} \Lambda\left(g^{-1} \operatorname{Exp}(t u) g\right) \\
& =\left.\frac{d}{d t}\right|_{t=0}\left(\Lambda(g)^{-1} \Lambda(\operatorname{Exp}(t u)) \Lambda(g)\right) \\
& =\left.\frac{d}{d t}\right|_{t=0} \Lambda(\operatorname{Exp}(t u)) \\
& =D_{e} \Lambda(u)
\end{aligned}
$$

so that $\lambda:=D_{e} \Lambda$ is Ad-invariant and hence a trace on the Lie algebra.

In order to associate a determinant map with a trace, we need the following preliminary result. We set

$$
\mathcal{P G}:=\left\{\gamma \in C^{1}([0,1], \mathcal{G}), \quad \gamma(0)=e_{\mathcal{G}}\right\} .
$$

Proposition 2 Let $\mathcal{G}$ be a Lie group and let $\lambda$ be a (resp. continuous) linear form on its Lie algebra. If the image of the fundamental group $\pi_{1}(\mathcal{G})$ under the map

$$
\begin{aligned}
\operatorname{Det}_{\lambda}: \mathcal{P G} & \rightarrow \mathbb{C}^{*} \\
\gamma & \mapsto \Lambda(\gamma(1))=e^{\int_{0}^{1} \lambda\left(L_{\gamma}-1\right)_{*} \dot{\gamma}}
\end{aligned}
$$

is trivial, then Det $_{\lambda}$ induces a map

$$
\begin{aligned}
\operatorname{det}_{\lambda}: \tilde{\mathcal{G}} & \rightarrow \mathbb{C}^{*} \\
g & \mapsto \operatorname{det}_{\lambda}(g):=\operatorname{Det}_{\lambda}([\gamma])
\end{aligned}
$$

where $\gamma$ is any path in $\mathcal{P G}$ such that $\gamma(1)=g$.

$\operatorname{det}_{\lambda}$ is differentiable at $e \in \mathcal{G}$ (resp. of class $C^{1}$ on $\mathcal{G}$ ) with differential

$$
D_{e} \operatorname{det}_{\lambda}=\lambda \text {. }
$$

Moreover, whenever $\lambda$ is Ad-invariant, then $\operatorname{det}_{\lambda}$ is multiplicative.

Proof: Since Det $_{\lambda}$ only depends on the homotopy class of $\gamma$, it induces a map $\operatorname{det}_{\lambda}$ on $\tilde{\mathcal{G}}$.

The map $\operatorname{det}_{\lambda}$ is differentiable at $e_{\mathcal{G}}$. Indeed, let $g(s)$ be a local one parameter 
local subgroup of $\mathcal{G}$ generated by $u \in \operatorname{Lie}(\mathcal{G})$. Then $t \mapsto \gamma_{g(s)}(t):=g(s t) \in \mathcal{P G}$ with $t \in[0,1]$, is a path that ends at $g(s)$ and

$$
\begin{aligned}
\frac{d}{\left.d s\right|_{s=0}} \operatorname{det}_{\lambda}(g(s)) & =\frac{d}{\left.d s\right|_{s=0}} \lambda\left(\left(L_{\gamma_{g(s)}^{-1}}\right)_{*}\left(\dot{\gamma}_{g(s)}\right)\right) \\
& =\frac{d}{\left.d s\right|_{s=0}} \lambda(s u) \\
& =\lambda(u)
\end{aligned}
$$

which shows that $D_{e} \operatorname{det}_{\lambda}=\lambda$.

Let us prove the multiplicativity of $\operatorname{det}_{\lambda}$ under the assumption that $\lambda$ is Adinvariant. Let $\gamma_{1}, \gamma_{2} \in \mathcal{P G}$ then

$$
\begin{aligned}
\lambda\left(\left(L_{\left(\gamma_{1} \gamma_{2}\right)^{-1}}\right)_{*} \frac{d}{d t}\left(\gamma_{1} \gamma_{2}\right)\right) & =\lambda\left(\left(L_{\gamma_{2}^{-1}}\right)_{*} \circ\left(L_{\gamma_{1}^{-1}}\right)_{*} \circ\left(L_{\gamma_{1}}\right)_{*}\left(\dot{\gamma}_{2}\right)\right) \\
& +\lambda\left(\left(L_{\left(\gamma_{1} \gamma_{2}\right)^{-1}}\right)_{*} \circ\left(R_{\gamma_{2}}\right)_{*}\left(\dot{\gamma}_{1}\right)\right) \\
& =\lambda\left(\left(L_{\gamma_{2}^{-1}}\right)_{*}\left(\dot{\gamma}_{2}\right)\right)+\lambda\left(A d_{\gamma_{2}} \circ\left(\left(L_{\gamma_{1}^{-1}}\right)_{*}\left(\dot{\gamma}_{1}\right)\right)\right)+ \\
& =\lambda\left(L_{\gamma_{2}^{-1}}\left(\dot{\gamma}_{2}\right)\right)+\lambda\left(L_{\gamma_{1}^{-1}}\left(\dot{\gamma}_{1}\right)\right),
\end{aligned}
$$

where we have used the Ad-invariance of $\lambda$ in the second identity. Integrating over $[0,1]$ yields the multiplicativity of $\operatorname{det}_{\lambda}$.

Definition 3 We call $\operatorname{det}_{\lambda}$ the determinant map on the Lie group associated with the trace $\lambda$ on its Lie algebra.

Summing up the above results in the case of a Lie group with exponential mapping we get:

Theorem 3 Let $\mathcal{G}$ be a Lie group with an exponential mapping $\operatorname{Exp}:$ Lie $(\mathcal{G}) \rightarrow$ $\mathcal{G}$.

1. To an Ad-invariant linear map (or equivalently to a trace if LieG $\subset \mathcal{G}$ ) $\lambda$ on its Lie algebra such that the image of the fundamental group $\pi_{1}(\mathcal{G})$ under the map

$$
\begin{aligned}
\operatorname{Det}_{\lambda}: \mathcal{P G} & \rightarrow \mathbb{C}^{*} \\
\gamma & \mapsto \Lambda(\gamma(1))=e^{\int_{0}^{1} \lambda\left(\left(L_{\gamma^{-1}}\right)_{*} \dot{\gamma}\right)}
\end{aligned}
$$

is trivial, corresponds a determinant map $\operatorname{det}_{\lambda}: \tilde{\mathcal{G}} \rightarrow \mathbb{C}^{*}$. It makes the following diagramm commute:

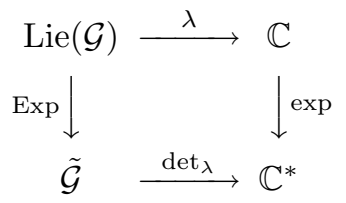

where exp is the ordinary exponential on complex numbers. 
2. Conversely, let $\Lambda: \tilde{\mathcal{G}} \rightarrow \mathbb{C}^{*}$ be a determinant map of class $C^{1}$. Then its differential

$$
\lambda:=D_{e} \Lambda
$$

is a continuous Ad-invariant map (and hence a continuous trace) and

$$
\Lambda=\operatorname{det}_{\lambda},
$$

so that the following diagram commutes:

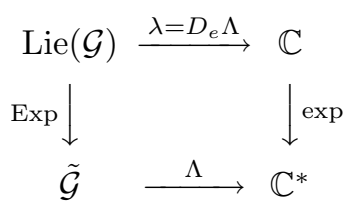

Proof: We first observe that $\operatorname{Exp}(\operatorname{Lie}(\mathcal{G})) \subset \tilde{\mathcal{G}}$ so that by the above lemma $\operatorname{det}_{\lambda}$ is well-defined on $\operatorname{Exp}(\operatorname{Lie}(\mathcal{G}))$. By Proposition 2, setting $\gamma_{u}(t):=\operatorname{Exp}(t u)$ we have:

$$
\begin{aligned}
\operatorname{det}_{\lambda}(\operatorname{Exp}(u)) & =\operatorname{det}_{\lambda}\left(\gamma_{u}(1)\right) \\
& =\exp \left(\lambda\left(\int_{0}^{1}\left(L_{\gamma_{u}(t)^{-1}}\right)_{*}\left(\dot{\gamma}_{u}(t)\right)\right)\right) \\
& =\exp (\lambda(u))
\end{aligned}
$$

so that the diagram commutes. Conversely, by Proposition 1, the multiplicativity of $\Lambda$ implies the Ad-invariance of $\lambda$ and hence its traciality. Since $D_{e} \Lambda=\lambda$, for any $g=\gamma(1) \in \tilde{\mathcal{G}}$ with $\gamma \in \mathcal{P G}$, by the continuity of $\lambda$ we have

$$
\begin{aligned}
\Lambda(g) & =\Lambda(\gamma(1)) \\
& =\exp \left(D_{e} \Lambda\left(\int_{0}^{1}\left(L_{\gamma(t)^{-1}}\right)_{*}(\dot{\gamma}(t))\right)\right) \\
& =\exp \left(\int_{0}^{1} \lambda\left(\left(L_{\gamma(t)^{-1}}\right)_{*}(\dot{\gamma}(t))\right)\right) \\
& =\operatorname{det}_{\lambda}(g),
\end{aligned}
$$

as a consequence of which the diagram commutes by the first part of the theorem

Example 1 Let $\mathcal{G}=G L_{n}(\mathbb{C})$ with Lie algebra Lie $(\mathcal{G})=g l_{n}(\mathbb{C})$ equipped with the usual trace $\lambda: g l_{n}(\mathbb{C}) \rightarrow \mathbb{C}, \lambda(A)=\operatorname{tr}(A)$ on matrices. Let $p: \mathbb{C}^{n} \rightarrow \mathbb{C}^{n}$ denote a projection, i.e. $p^{2}=p$. Setting $V_{0}=\operatorname{Ker} p, V_{1}:=\operatorname{Im} p$, we have $V_{0} \oplus V_{1}=\mathbb{C}^{n}$ so that for $u=u_{0}+u_{1} \in V_{0} \oplus V_{1}$, we have $e^{2 i \pi p} u=u_{0}+e^{2 i \pi} u_{1}=$ u. One can show (see e.g. [Ka]) that the fundamental group $\pi_{1}\left(G L_{n}(\mathbb{C})\right.$ ) is generated by the homotopy classes $[l]$ of the loops $l(t)=e^{2 i \pi t p}$ where $p: \mathbb{C}^{n} \rightarrow$ $\mathbb{C}^{n}$ is a projector. Since $\operatorname{Det}_{\lambda}(l)=e^{2 i \pi \int_{0}^{1} \lambda(p)}=e^{2 i \pi(\operatorname{rk}(p))}$ where $\operatorname{rk}(p) \in \mathbb{N}$ is the rank of $p$, it follows that the image by $\lambda$ of $\pi_{1}\left(G L_{n}(\mathbb{C})\right)$ is trivial. $\operatorname{det}_{\lambda}$ built this way corresponds to the usual determinant on invertible matrices. 


\section{Traces on zero order classical pseudodifferen- tial operators}

\subsection{A Lie group structure on invertible zero order classical pseudodifferential operators}

Let $M$ be an $n$-dimensional closed Riemannian manifold and $E \rightarrow M$ a smooth vector bundle based on $M$ with rank $k$. For $m \in \mathbb{C}$, the linear space $C \ell^{m}(M, E)$ of classical pseudodifferential operators of order $m$ can be equipped with a Fréchet topology. For this, one first equips the set $C S^{m}(U, W)$ of classical symbols of order $m$ on an open subset $U$ of $\mathbb{R}^{n}$ with values in an euclidean vector space $W$ (with norm $\|\cdot\|$ ) with a Fréchet structure. Such a symbol reads:

$$
\sigma=\sum_{j=0}^{N-1} \psi \sigma_{m-j}+\sigma_{(N)},
$$

where $\psi$ is a smooth function which vanishes at 0 and equals to one outside a compact, where $\sigma_{m-j} \in C^{\infty}\left(S^{*} U, \operatorname{End}(W)\right)$ is positively homogeneous in $\xi$ of order $m-j$ and where $\sigma_{(N)} \in C^{\infty}\left(S^{*} U, \operatorname{End}(W)\right)$ is a symbol of order $m-N$. The following semi-norms labelled by multiindices $\alpha, \beta$ and integers $j \geq 0, N$ give rise to a Fréchet topology on $C S^{m}(U, W)[\mathrm{H}]$ (see also $\left.[\mathrm{Po}]\right)$ :

$$
\begin{aligned}
& \sup _{x \in K, \xi \in \mathbb{R}^{n}}(1+|\xi|)^{-\operatorname{Re}(m)+|\beta|}\left\|\partial_{x}^{\alpha} \partial_{\xi}^{\beta} \sigma(x, \xi)\right\| ; \\
& \sup _{x \in K, \xi \in \mathbb{R}^{n}}(|\xi|)^{-\operatorname{Re}(m)+N+|\beta|}\left\|\partial_{x}^{\alpha} \partial_{\xi}^{\beta}\left(\sigma-\sum_{j=0}^{N-1} \psi(\xi) \sigma_{m-j}\right)(x, \xi)\right\| ; \\
& \sup _{x \in K,|\xi|=1}\left\|\partial_{x}^{\alpha} \partial_{\xi}^{\beta} \sigma_{m-j}(x, \xi)\right\|
\end{aligned}
$$

where $K$ is any compact set in $U$.

Let $C S^{-\infty}(U, W):=\bigcap_{m \in \mathbb{R}} C S^{m}(U, W)$ corresponds to the algebra of smoothing symbols.

Any given cut-off function $\psi$ induces a map:

$$
\begin{aligned}
C S^{m}(U, W) & \rightarrow C S^{m}(U, W) / C S^{m-N}(U, W) \oplus C S^{m-N}(U, W) \\
\sigma & \mapsto\left(\sum_{j=0}^{N} \sigma_{m-j} ; \sigma-\sum_{j=0}^{N} \psi \sigma_{m-j}\right)
\end{aligned}
$$

If we equip $C S^{m}(U, W) / C S^{m-N}(U, W)$ with the topology of $\left(C^{\infty}\left(S^{*} U, W\right)\right)^{N}$ and $C S^{m}(U, W)$ with the ordinary topology of symbols namely

$$
\sup _{x \in K, \xi \in \mathbb{R}^{n}}(1+|\xi|)^{-\operatorname{Re}(m)+|\beta|}\left\|\partial_{x}^{\alpha} \partial_{\xi}^{\beta} \sigma(x, \xi)\right\|,
$$

then $C S^{m}(U, W)$ appears as the projective limit of the following system:

$$
\left(C S^{m}(U, W) / C S^{m-N} \oplus C S^{m-N} ; \pi_{N}\right)_{N \geq 0},
$$

where

$$
\begin{aligned}
\pi_{N}: C S^{m}(U, W) / C S^{m-N} \oplus C S^{m-N} & \rightarrow C S^{m}(U, W) / C S^{m-N+1} \oplus C S^{m-N+1} \\
\left(\sum_{j=0}^{N} \sigma_{m-j}, \eta\right) & \mapsto\left(\sum_{j=0}^{N+1} \sigma_{m-j}, \eta+\psi \sigma_{m-j-N-1}\right) .
\end{aligned}
$$


Accordingly, $C S^{m}(U, W) / C S^{-\infty}(U, W)$ is the topological projective limit of the system $C S^{m}(U, W) / C S^{m-N}(U, W), N \in \mathbb{N}_{0}$ that is the linear space of formal power series with coefficients in $C^{\infty}\left(S^{*} U, W\right)$ :

$$
C S^{m}(U, W) / C S^{-\infty}(U, W) \simeq h^{m} C^{\infty}\left(S^{*} U, W\right)\left[\left[h^{-1}\right]\right] .
$$

Given a vector bundle based on a closed manifold $M$, the set $C \ell^{m}(M, E)$ of classical pseudodifferential operators acting on sections of $E$, namely pseudodifferential operators $A$ acting on sections of $E$ that have local classical symbols $\sigma_{A}^{U} \in C S(U, W)$ in a local trivialization $E_{\left.\right|_{U}} \simeq U \times W$. , inherits a Fréchet structure via the Fréchet structure on classical symbols. Given an atlas $\left(U_{i}, \phi_{i}\right)_{i \in I}$ on $M$ and corresponding local trivializations $E_{\left.\right|_{i}} \simeq U_{i} \times F$ where $F$ is the model fibre of $E$, in any local chart $U_{i}$, we can equip $C \ell^{m}(M, E)$ with the following family of seminorms labelled by multiindices $\alpha, \beta$ and integers $j \geq 0, i \in I$, $N \geq 0$

$$
\begin{aligned}
& \sup _{x \in K, \xi \in \mathbb{R}^{n}}(1+|\xi|)^{-\operatorname{Re}(m)+|\beta|}\left\|\partial_{x}^{\alpha} \partial_{\xi}^{\beta} \sigma_{A}^{(i)}(x, \xi)\right\| ; \\
& \sup _{x \in K, \xi \in \mathbb{R}^{n}}(|\xi|)^{-\operatorname{Re}(m)+N+|\beta|}\left\|\partial_{x}^{\alpha} \partial_{\xi}^{\beta}\left(\sigma_{A}^{(i)}-\sum_{j=0}^{N-1} \psi(\xi)\left(\sigma_{A}^{(i)}\right)_{m-j}\right)(x, \xi)\right\| ; \\
& \sup _{x \in K,|\xi|=1}\left\|\partial_{x}^{\alpha} \partial_{\xi}^{\beta}\left(\sigma_{A}^{(i)}\right)_{m-j}(x, \xi)\right\|,
\end{aligned}
$$

where $K$ is any compact subset of $\phi_{i}\left(U_{i}\right) \subset \mathbb{R}^{n}$.

The algebra of integer order operators

$$
C \ell^{\mathbb{Z}}(M, E):=\bigcup_{m \in \mathbb{Z}} C \ell^{m}(M, E)
$$

is equipped with the inductive limit topology of Fréchet spaces. It is strictly contained in the whole algebra of classical pseudodifferential operators $C \ell(M, E)$ generated by $\bigcup_{m \in \mathbb{R}} C \ell^{m}(M, E)$. We shall also consider the group

$$
C \ell^{*}(M, E)=\left\{A \in C \ell(M, E), \quad \exists A^{-1} \in C \ell(M, E)\right\}
$$

of invertible classical pseudodifferential operators.

Remark 3 When $E$ is the trivial bundle $M \times \mathbb{R}$, we drop $E$ in the notation writing $C \ell^{m}(M)$ instead of $C \ell^{m}(M, E), C \ell^{\mathbb{Z}}(M)$ instead of $C \ell^{\mathbb{Z}}(M, E), C \ell^{*}(M)$ instead of $C \ell^{*}(M, E)$.

Setting $m=0$ endows the class $C \ell^{0}(M, E)$ of zero order classical pseudodifferential operators with a Fréchet Lie algebra structure. Let

$$
C \ell^{0, *}(M, E):=\left\{A \in C \ell^{0}(M, E), \quad \exists A^{-1} \in C \ell^{0}(M, E)\right\}
$$

be the group of invertible zero order classical pseudodifferential operators which is strictly contained in the intersection $C \ell^{0}(M, E) \cap C \ell^{*}(M, E)$.

Remark 4 It is useful to note that $C \ell^{*}(M, E)$ acts on $C \ell^{m}(M, E)$ for any $m \in \mathbb{R}$ by the adjoint action defined for $P \in C \ell^{*}(M, E)$ by

$$
\begin{aligned}
C \ell^{m}(M, E) & \rightarrow C \ell^{m}(M, E) \\
A & \mapsto A d_{P} A:=P^{-1} A P
\end{aligned}
$$

and specifically on the algebras $C \ell^{\mathbb{Z}}(M, E)$ and $C \ell^{0}(M, E)$. 
Proposition $3[K V 2] C \ell^{0, *}(M, E)$ is a Fréchet Lie group with exponential map and its Lie alegbra is $C \ell^{0}(M, E)$.

Proof: As an open subset in the Fréchet space $C \ell^{0}(M, E), C \ell^{0, *}(M, E)$ is a Fréchet manifold modelled on $C \ell^{0}(M, E)$.

Since $\sigma_{A B} \sim \sigma_{A} \star \sigma_{B}$ for two operators $A, B$ with symbols $\sigma_{A}, \sigma_{B}$, the smoothness of the product map on $C \ell(M, E)$ follows from the smoothness of the symbol product $\star$ on $C S(U, W)$ for any vector space $W$ :

$$
\sigma \star \tau \sim \sum_{\alpha} \frac{1}{\alpha !} \partial_{\xi}^{\alpha} \sigma \partial_{x}^{\alpha} \tau .
$$

Similarly, the smoothness of the inversion $A \mapsto A^{-1}$ on $C \ell^{0, *}(M, E)$ follows from the fact that for an operator $A \in C \ell^{0, *}(M, E)$ of order $m$ with symbol $\sigma_{A}$,

$$
\left(\sigma_{A^{-1}}\right)_{-m-j}=\frac{i}{2 \pi} \int_{\Gamma} \lambda^{-1} \tau_{-m-j}(A) d \lambda
$$

where $\Gamma$ is a contour around the spectrum of $A$ and where

$$
\begin{aligned}
\tau_{-m}(A) & =\left(\sigma_{A}-\lambda\right)^{-1} \\
\tau_{-m-j}(A) & =-\tau_{-m}(A) \sum_{k+l+|\alpha|=j, l, j} i^{-|\alpha|} \frac{1}{\alpha !} \partial_{\xi}^{\alpha}\left(\sigma_{A}\right)_{m-k} \partial_{x}^{\alpha} \tau_{-m-l}(A) .
\end{aligned}
$$

Hence $C \ell^{0, *}(M, E)$ is a Fréchet Lie group with Fréchet Lie algebra $C \ell^{0}(M, E)$. Given any $B \in C \ell^{0}(M, E)$, the first order differential equation:

$$
A_{t}^{-1} \dot{A}_{t}=B, \quad A_{0}=I
$$

has a unique solution in $C \ell^{0, *}(M, E)$ given by

$$
A_{t}=\frac{i}{2 \pi} \int_{\Gamma} \exp (t \lambda)(B-\lambda)^{-1} d \lambda
$$

where exp is the ordinary exponential on complex numbers and where $\Gamma$ is as before a contour around the spectrum of $B$. This defines an exponential map

$$
\operatorname{Exp}: C \ell^{0}(M, E) \rightarrow C \ell^{0, *}(M, E)
$$

and ends the proof.

Let $C \ell^{-\infty}(M, E):=\bigcap_{m \in \mathbb{R}} C \ell^{m}(M, E)$ (we write $C \ell^{-\infty}(M)$ if $E=M \times \mathbb{C}$ ) denote the class of smoothing operators acting on sections of $E$ then clearly

$$
C \ell^{m}(M, E) / C \ell^{-\infty}(M, E) \simeq h^{m} C^{\infty}\left(S^{*} M, \pi^{*} E\right)\left[\left[h^{-1}\right]\right]
$$

and hence

$$
C \ell^{\mathbb{Z}}(M, E) / C \ell^{-\infty}(M, E) \simeq C^{\infty}\left(S^{*} M, p^{*} E\right)\left[\left[h^{-1}, h\right],\right.
$$

where $\pi^{*} E$ is the pull-back of the vector bundle $E$ by the canonical projection $p: S^{*} M \rightarrow M$, and for any ring $R: R[[x]]=\left\{\sum_{j=0}^{+\infty} r_{j} x^{j} \mid r_{j} \in R\right\} . R\left[\left[x, x^{-1}\right]=\right.$ $\left\{\sum_{j=-k}^{+\infty} r_{j} x^{j} \mid r_{j} \in R, k \in \mathbb{Z}\right\}$. 


\subsection{Hochschild homology on the algebra of zero order clas- sical pseudodifferential operators}

Let $\mathcal{A}$ be an associative (resp. topological) algebra over $\mathbb{R}$ with unit 1 . The space $C^{k}(\mathcal{A})$ of scalar valued (resp. continuous) $k+1$-linear forms on $\mathcal{A}$ corresponds to the space of $n$-cochains on $\mathcal{A}$. The Hochschild coboundary is the map:

$$
\begin{aligned}
C^{k}(\mathcal{A}) & \rightarrow C^{k+1}(\mathcal{A}) \\
\chi & \mapsto b \chi
\end{aligned}
$$

defined by

$$
\begin{aligned}
(b \chi)\left(a_{0}, \cdots, a_{k+1}\right) & =\sum_{j=0}^{k}(-1)^{j} \chi\left(a_{0}, \cdots, a_{j} a_{j+1}, \cdots, a_{k+1}\right) \\
& +(-1)^{k+1} \chi\left(a_{k+1} a_{0}, \cdots, a_{k}\right) .
\end{aligned}
$$

It satisfies the condition $b^{2}=0$ and hence defines a cohomology $H H^{*}(\mathcal{A})$ (resp. $\overline{H H}^{*}(\mathcal{A})$ ), the Hochschild cohomology of $\mathcal{A}$. In particular, $H H^{0}(\mathcal{A})$ (resp. $\left.\overline{H H}_{0}^{*}(\mathcal{A})\right)$ corresponds to (resp. continuous) traces on $\mathcal{A}$, i.e. (resp. continuous) linear maps $\chi$ on $\mathcal{A}$ with vanishing coboundary: $\forall a, b \in A, \chi(a b)=\chi(b a)$.

On the other hand the space of $k$-chains

$$
C_{k}(\mathcal{A})=\mathcal{A} \otimes \mathcal{A} \otimes \cdots \otimes \mathcal{A} \quad(k+1 \text { factors })
$$

(resp.

$$
\left.C_{k}(\mathcal{A})=\mathcal{A} \hat{\otimes}_{\pi} \mathcal{A} \hat{\otimes}_{\pi} \cdots \hat{\otimes}_{\pi} \mathcal{A} \quad(k+1 \text { factors })\right)
$$

where $\otimes\left(\right.$ resp. $\left.\hat{\otimes}_{\pi}\right)$ denotes the (projective completion of) the tensor product, is equipped with (the extension to this completion of) the Hochschild boundary map $b: C_{k}(\mathcal{A}) \rightarrow C_{k-1}(\mathcal{A})$ defined by

$b\left(a_{0} \otimes a_{1} \cdots \otimes a_{k}\right)=\sum_{i=0}^{k-1}(-1)^{i} a_{0} \otimes \cdots \otimes a_{i} a_{i+1} \otimes \cdots \otimes a_{k}+(-1)^{k} a_{k} a_{0} \otimes \cdots \otimes a_{k-1}$.

Similarly, it satisfies the condition $b^{2}=0$ and hence defines a homology $H H_{*}(\mathcal{A})$ (resp. $\left.\overline{H H}_{*}(\mathcal{A})\right)$, the Hochschild homology of $\mathcal{A}$ and it is easy to check that $H H^{0}(\mathcal{A})$ (resp. $\overline{H H}^{0}(\mathcal{A})$ ) is isomorphic to the (resp. topological) dual of $H H_{0}(\mathcal{A})\left(\operatorname{resp} . \overline{H H}_{0}(\mathcal{A})\right)$.

Lemma 3 The space of (resp. continuous) traces on $C \ell^{0}(M, E)$ is isomorphic to the (resp. topological) dual of the zeroth homology group $H H_{0}\left(C \ell^{0}(M) / C \ell^{-\infty}(M)\right)$ (resp. $\left.\overline{H H}_{0}\left(C \ell^{0}(M) / C \ell^{-\infty}(M)\right)\right)$.

Proof: Since the proof in the continuous case goes as in the algebraic case, we only discuss the latter. The space $H H^{0}\left(C \ell^{0}(M, E)\right)$ of traces on $C \ell^{0}(M, E)$ is isomorphic to the dual of the zeroth homology group $H H_{0}\left(C \ell^{0}(M, E)\right)$. Fixing an idempotent $p \in g l_{N}\left(C^{\infty}(M)\right)$ such that $E=p\left(M \times \mathbb{C}^{N}\right)$ with $N$ a positive integer chosen large enough, yields a Morita equivalence between the algebras $C \ell^{0}(M, E)$ and $C \ell^{0}(M)$ and provides natural isomorphisms between 
their Hochschild homology groups (the trace on $E$ induces such an isomorphism with inverse equal to the natural inclusion) so that

$$
H H_{0}\left(C \ell^{0}(M, E)\right) \simeq H H_{0}\left(C \ell^{0}(M)\right) .
$$

On the other hand, M. Wodzicki proved in [W88] that the Hochschild homology groups of $C \ell^{0}(M)$ and $C \ell^{0}(M) / C \ell^{-\infty}(M)$ coincide except perhaps in degree 1 . As a consequence

$$
H H_{0}\left(C \ell^{0}(M, E)\right) \simeq H H_{0}\left(C \ell^{0}(M) / C \ell^{-\infty}(M)\right)
$$

and the result of the lemma follows.

When $\mathcal{A}$ is the locally convex topological algebra $C^{\infty}(X)$ with $X$ a smooth manifold, then the Hochschild-Kostant-Rosenberg (HKR) map extended to $C^{\infty}(X)$ by Connes [Co]

$$
\begin{aligned}
\beta: C_{k}\left(C^{\infty}(X)\right) & \rightarrow \Omega^{k}(X) \\
a_{0} \otimes \cdots \otimes a_{k} & \mapsto a_{0} d a_{1} \wedge \cdots \wedge d a_{k},
\end{aligned}
$$

yields, when $X$ is compact, a canonical isomorphism (resp. of topological spaces) between $H H_{k}\left(C^{\infty}(X)\right)\left(\right.$ resp. $\left.\overline{H H}_{k}\left(C^{\infty}(X)\right)\right)$ and the space $\Omega^{k}(X)$ of $k$-forms on $X$.

Let us set

$$
\mathcal{H}_{0}:=C^{\infty}\left(S^{*} M\right)\left[\left[h^{-1}\right]\right]=\mathbb{C}\left[\left[h^{-1}\right]\right] \otimes C^{\infty}\left(S^{*} M\right)
$$

equipped with the product of formal power series and

$$
\mathcal{H}:=\bigcup_{m \in \mathbb{Z}} h^{m} \mathcal{H}_{0}=C^{\infty}\left(S^{*} M\right)\left[\left[h^{-1}, h\right]=\mathbb{C}\left[\left[h^{-1}, h\right] \otimes C^{\infty}\left(S^{*} M\right)\right.\right.
$$

equipped with the inductive limit topology.

Similarly we set $\mathcal{A}_{0}:=C \ell^{0}(M) / C \ell^{-\infty}(M)$ equipped with the operator product and $\mathcal{A}:=C \ell^{\mathbb{Z}}(M) / C \ell^{-\infty}(M)$ with $C \ell^{\mathbb{Z}}(M)$ equipped with the inductive limit topology of Fréchet spaces.

Recall from equation (3) the following isomorphisms of topological vector spaces:

$$
\mathcal{A}_{0} \simeq \mathcal{H}_{0} ; \quad \mathcal{A} \simeq \mathcal{H}
$$

With these isomorphisms, we have

$$
C_{k}\left(\mathcal{A}_{0}\right) \simeq C^{\infty}\left(S^{*} M^{k+1}\right)\left[\left[h_{0}^{-1}, \ldots, h_{k}^{-1}\right]\right] \simeq \otimes^{k} \mathcal{H}_{0}
$$

with the ordinary tensor product replaced by $\hat{\otimes}_{\pi}^{k}$ in the continuous case.

Lemma 4 The HKR-isomorphism holds for $\mathcal{H}_{0}$ and $\mathcal{H}$, that is, the map (6) with $X=T^{*} M \backslash M$ restricted to the subcomplexes $C_{*}\left(\mathcal{H}_{0}\right)$ and $C_{*}(\mathcal{H})$ induces isomorphisms:

$$
\beta: H H_{k}\left(\mathcal{H}_{0}\right) \stackrel{\simeq}{\rightarrow} \Omega^{k}\left(\mathcal{H}_{0}\right) ; \quad \beta: H H_{k}(\mathcal{H}) \stackrel{\simeq}{\rightarrow} \Omega^{k}(\mathcal{H}),
$$

and isomorphisms of topological spaces:

$$
\bar{\beta}: \overline{H H}_{k}\left(\mathcal{H}_{0}\right) \stackrel{\cong}{\rightrightarrows} \Omega^{k}\left(\mathcal{H}_{0}\right) ; \quad \bar{\beta}: \quad \overline{H H}_{k}(\mathcal{H}) \stackrel{\cong}{\rightrightarrows} \Omega^{k}(\mathcal{H} .
$$


Proof : We carry out the proof in the algebraic setting since it extends in a straightoforward way to the topological setting. Since $\mathcal{H}_{0} \simeq \mathbb{C}\left[\left[h^{-1}\right]\right] \otimes$ $C^{\infty}\left(S^{*} M\right)$ and since HKR-isomorphisms hold for both $\mathbb{C}\left[\left[h^{-1}\right]\right]$ and $C^{\infty}\left(S^{*} M\right)$, we get by the Künneth formula ([Lo]):

$H H_{*}\left(\mathcal{H}_{0}\right) \stackrel{\mathrm{sh}^{-1}}{\longrightarrow} H H_{*}\left(\mathbb{C}\left[\left[h^{-1}\right]\right]\right) \otimes H H_{*}\left(C^{\infty}\left(S^{*} M\right)\right) \stackrel{\mathrm{HKR}}{\longrightarrow} \Omega^{*}\left(\mathbb{C}\left[\left[h^{-1}\right]\right]\right) \otimes \Omega^{*}\left(S^{*} M\right) \stackrel{\wedge}{\longrightarrow} \Omega^{*}\left(\mathcal{H}_{0}\right)$.

where sh denotes the shuffle product in Hochschild homology ([Lo]), and the composition is equal to the map induced by $\bar{\beta}$. A similar proof holds for $H H_{*}(\mathcal{H})$ replacing $C\left[\left[h^{-1}\right]\right]$ by $C\left[\left[h^{-1}, h\right]\right.$.

Both $H H_{*}(\mathcal{H}) \simeq \Omega^{*}(\mathcal{H})\left(\right.$ resp. $\left.\overline{H H}_{*}(\mathcal{H}) \simeq \Omega^{*}(\mathcal{H})\right)$ and $H H_{*}\left(\mathcal{H}_{0}\right) \simeq \Omega^{*}\left(\mathcal{H}_{0}\right)$ (resp. $\left.\overline{H H}_{*}\left(\mathcal{H}_{0}\right) \simeq \Omega^{*}\left(\mathcal{H}_{0}\right)\right)$ have natural gradings described through the HKR isomorphism as follows:

$$
\begin{aligned}
\Omega^{0}\left(\mathcal{H}_{0}\right)[p] & =\Omega^{0}(\mathcal{H})[p]=h^{p} C^{\infty}\left(S^{*} M\right) \\
\Omega^{k}\left(\mathcal{H}_{0}\right)[p] & =\Omega^{k}(\mathcal{H})[p]=h^{p}\left(\Omega^{k}\left(S^{*} M\right) \oplus \frac{d h}{h} \wedge \Omega^{k-1}\left(S^{*} M\right)\right) \text { if } p<0, \text { and } k>0 \\
\Omega^{k}(\mathcal{H})[0] & =\Omega^{k}\left(S^{*} M\right) \oplus \frac{d h}{h} \wedge \Omega^{k-1}\left(S^{*} M\right) \text { if } k>0 \\
\Omega^{k}\left(\mathcal{H}_{0}\right)[0] & \left.=\left\{f \in \Omega^{k}(\mathcal{H})[0] \mid d h\right\lrcorner f=0\right\}=\Omega^{k}\left(S^{*} M\right)
\end{aligned}
$$

On the other hand the filtration on $\mathcal{A}_{0}$ given by the order i.e. for any $p \leq 0$ by (see equation (4))

$$
\mathcal{A}_{p}=C \ell^{p}(M) / C \ell^{-\infty}(M) \simeq h^{p} \mathcal{H}_{0}
$$

which therefore induces a filtration on the Hochschild complex given by :

$$
\begin{aligned}
& F_{p}\left(C_{k}\left(\mathcal{A}_{0}\right)\right)=\bigoplus_{\substack{\sum_{p_{j}=p ;} \\
p_{j} \leq 0}} \mathcal{A}_{p_{0}} \otimes \mathcal{A}_{p_{1}} \otimes \cdots \otimes \mathcal{A}_{p_{k}} \\
& \simeq \bigoplus_{\substack{\sum p_{j}=p ; \\
p_{j} \leq 0}} h_{0}^{p_{0}} \cdots h_{k}^{p_{k}} C^{\infty}\left(S^{*} M^{k+1}\right)\left[\left[h_{0}^{-1}, \ldots, h_{k}^{-1}\right]\right],
\end{aligned}
$$

where the direct sums need to be completed in the continuous case. Clearly the Hochschild map is compatible with this filtration.

Lemma 5 The filtration $\left(F_{p}\left(C_{*}\left(\mathcal{A}_{0}\right)\right)\right)_{p \in-\mathbb{N}}$ is complete, that is :

$$
C_{*}\left(\mathcal{A}_{0}\right) \simeq \lim _{\leftarrow} C_{*}(\mathcal{A}) / F_{p}\left(C_{*}\left(\mathcal{A}_{0}\right)\right) .
$$

Proof : This follows from the fact that we have algebras of formal series.

\subsection{A uniqueness result for traces on zero order classical pseudodifferential operators}

There are at least two known types of continuous traces on $C \ell^{0}(M, E)$.

- The Wodzicki residue [W], [K]:

$$
\operatorname{res}(A):=\frac{1}{(2 \pi)^{n}} \int_{M} d x \int_{|\xi|=1}\left(\operatorname{tr}_{x} \sigma_{A}(x, \xi)\right)_{-n} d_{S} \xi
$$


- Leading symbol traces [PR1], associated to any current $\tau \in C^{\infty}\left(S^{*} M\right)^{\prime}$ :

$$
\operatorname{Tr}_{0}^{\tau}(A):=\tau\left(\left(\operatorname{tr}_{x}\left(\sigma_{A}(x, \xi)\right)\right)_{0}\right) .
$$

Whenever $\tau(1) \neq 0$ we set:

$$
\operatorname{tr}_{0}^{\tau}(A):=\frac{\operatorname{Tr}_{0}^{\tau}(A)}{\tau(1)}=\frac{\tau\left(\left(\operatorname{tr}_{x}\left(\sigma_{A}(x, \xi)\right)\right)_{0}\right)}{\tau(1)} .
$$

Here $(\tau(x, \xi))_{j}$ denotes the positively homogeneous part of the symbol $\tau(x, \xi)$ of degree $j$ and $d_{S} \xi=\sum_{i=1}^{n}(-1)^{i+1} \xi_{i} d \xi_{1} \wedge \cdots \wedge d \hat{\xi}_{i} \wedge \cdots \wedge d \xi_{n}$ is the volume form on the unit cosphere at the point $x$.

Remark 5 - Their continuity in the Fréchet topology of $C \ell^{0}(M, E)$ follows from the fact that they are defined in terms of a finite number of homogeneous parts of the symbols. The Wodzicki residue extends to the unique (up to a multiplicative factor and when $\operatorname{dim} M>1$ ) trace on $C \ell^{\mathbb{Z}}(M, E)$ which is also continuous but the leading symbol trace does not.

- The Wodzicki residue and leading symbol trace are closely related. Indeed, from the Wodzicki residue and an operator $Q \in C \ell^{*}(M, E)$ with non zero order $q$ and scalar leading symbol, one can build a leading symbol trace on $C \ell^{0}(M, E)$ as follows:

$$
\operatorname{res}_{0}^{Q}: A \mapsto \operatorname{res}\left(A Q^{-\frac{n}{q}}\right) .
$$

It reads:

$$
\begin{aligned}
\operatorname{res}_{0}^{Q}(A) & =\frac{1}{(2 \pi)^{n}} \int_{S^{*} M} d x d_{S} \xi \operatorname{tr}_{x}\left(\sigma_{-n}\left(A Q^{-\frac{n}{q}}\right)\right) \\
& =\frac{1}{(2 \pi)^{n}} \int_{S^{*} M} d x d_{S} \xi \sigma_{-n}\left(Q^{-\frac{n}{q}}\right) \operatorname{tr}_{x}\left(\sigma_{0}(A)\right) \\
& =\frac{1}{(2 \pi)^{n}} \operatorname{TR}_{0}^{\tau^{Q}}(A),
\end{aligned}
$$

with $\tau^{Q}(A)(f)=\frac{1}{(2 \pi)^{n}} \int_{S^{*} M} \sigma_{-n}\left(Q^{-\frac{n}{q}}\right) f d x d_{S} \xi$. In particular, on a spin manifold $M$, letting $E$ be the spinor bundle on $M$ and choosing $Q=$ $|D|$ with $D$ the Dirac operator, we get

$$
\operatorname{res}_{0}^{|D|}(A)=\operatorname{res}\left(A|D|^{-n}\right)=\frac{1}{(2 \pi)^{n}} \int_{S^{*} M} d x d_{S} \xi \operatorname{tr}_{x}\left(\sigma_{0}(A)\right) .
$$

An important property is the invariance of these traces under the adjoint action of the whole group of invertible classical pseudodifferential oeprators.

Proposition 4 Both the Wodzicki residue and the leading symbol traces are invariant under the adjoint action of $C \ell^{*}(M, E)$.

Proof: This clearly holds for the Wodzicki residue since it extends to a trace on $C \ell(M, E)$. It also holds for the leading symbol traces since for any $C \in$ 
$C \ell^{*}(M, E)$ and any $A \in C \ell(M, E)$ we have:

$$
\begin{aligned}
\operatorname{Tr}_{0}^{\tau}\left(C^{-1} A C\right) & =\tau\left(\left(\operatorname{tr}_{x}\left(\sigma_{C^{-1} A C}(x, \xi)\right)\right)_{0}\right) \\
& =\tau\left(\operatorname{tr}_{x}\left(\sigma_{C^{-1} A C}^{L}(x, \xi)\right)\right) \\
& =\tau\left(\operatorname{tr}_{x}\left(\left(\sigma_{C}^{L}\right)^{-1} \sigma_{A}^{L} \sigma_{C}^{L}(x, \xi)\right)\right) \\
& =\tau\left(\operatorname{tr}_{x}\left(\sigma_{A}^{L}\right)\right) \\
& =\operatorname{Tr}_{0}^{\tau}(A)
\end{aligned}
$$

where $\sigma^{L}$ denotes the leading part of the symbol $\sigma$.

Remark 6 Leading symbol traces were introduced in [PR1] to define characteristic classes on infinite dimensional manifolds with structure group $C \ell_{0}^{*}(M, E)$. An operator $A \in C \ell^{0}(M, E)$ gives rise to functions of the operator

$$
f(A)=\frac{i}{2 \pi} \int_{\Gamma} f(\mu)(A-\mu)^{-1} d \mu
$$

with $f$ defined on the contour $\Gamma$ around the spectrum of $A$. Thus, a continuous trace $\lambda: C \ell^{0}(M, E) \rightarrow \mathbb{C}$ gives rise to functionals $A \mapsto \lambda(f(A))$ on $C \ell^{0}(M, E)$ that are invariant under the adjoint action of $C \ell_{0}^{*}(M, E)$ :

$$
\begin{aligned}
\lambda\left(f\left(C^{-1} A C\right)\right) & =\frac{i}{2 \pi} \int_{\Gamma} f(\mu) \lambda\left(\left(C^{-1} A C-\mu\right)^{-1}\right) d \mu \\
& =\frac{i}{2 \pi} \int_{\Gamma} f(\mu) \lambda\left(C(A-\mu)^{-1} C^{-1}\right) d \mu \\
& =\frac{i}{2 \pi} \int_{\Gamma} f(\mu) \lambda\left((A-\mu)^{-1}\right) d \mu \\
& =\frac{i}{2 \pi} \int_{\Gamma} f(\mu) \lambda\left((A-\mu)^{-1}\right) d \mu \\
& =\lambda(f(A)) .
\end{aligned}
$$

This holds in particular for polynomials $f(\mu)=P(\mu)$ with $P(X) \in \mathbb{C}[X]$ leading to invariant polynomials. When the structure group of the infinite manifold is $C \ell_{0}^{*}(M, E)$ and the curvature $\Omega$ of a connection on the manifold lies in $C \ell(M, E)$, such invariant polynomials lead to Chern-Weil characteristic classes $\lambda(P(\Omega))$ on the manifold.

The two traces res and $\operatorname{Tr}_{0}^{\tau}$ described above lead to two types of characteristic classes res $(P(\Omega))$ and $\operatorname{Tr}_{0}^{\tau}(P(\Omega))$ investigated in [PR1].

Let us illustrate the two types of traces with some examples.

Example 2 - Let $A: \sigma \mapsto a \sigma$ be a multiplication operator acting on sections of a vector bundle $E$ based on $M$ with a given by a section of End $\mathrm{E}$. Then $\operatorname{Tr}_{0}^{\tau}(A)=\tau\left(\operatorname{tr}_{x}(a)\right)$ so that for $\tau(f):=\int_{S^{*} M} f(x) d_{S} \xi d x$ we have

$$
\begin{aligned}
\operatorname{tr}_{0}^{\tau}(A) & =\frac{\int_{S^{*} M} \operatorname{tr}_{x}(a) d_{S} \xi d x}{\operatorname{Vol}\left(S^{*} M\right)} \\
& =\frac{\int_{M} \operatorname{tr}_{x}(a) d x}{\operatorname{Vol}(M)}
\end{aligned}
$$

where Vol denotes the volume. 
- Let $P$ be a pseudodifferential idempotent $P^{2}=P$, then its leading symbol $p \in C^{\infty}\left(T^{*} M\right)$ is also an idempotent so that the fibrewise $\operatorname{trace}^{\operatorname{tr}_{x}}(p(x, \cdot))$ is the $\operatorname{rank} \operatorname{rk}(p(x, \cdot)) \in \mathbb{N}$ which is constant in $(x, \xi) \in T^{*} M$ since $p$ is smooth. Hence

$$
\begin{aligned}
\operatorname{Tr}_{0}^{\tau}(P) & :=\tau\left(\left(\operatorname{tr}_{x} p\right)_{0}\right) \\
& =\operatorname{rk}(p) \operatorname{Tr}_{0}^{\tau}(1)
\end{aligned}
$$

As a consequence we have that

$$
\operatorname{tr}_{0}^{\tau}(P)=\operatorname{rk}(p) \in \mathbb{N}
$$

and in particular,

$$
\operatorname{tr}_{0}^{\tau}(1)=\operatorname{rk}(E)
$$

On the other hand

$$
\operatorname{res}(1)=0
$$

and more generally, the Wodzicki residue vanishes on zero order pseudodifferential projections [W84] (see also [Po]).

- We take this example from [Sc]. Let $M$ be a $n=2 m$-dimensional manifold and let $\Delta^{E}=\operatorname{tr}\left(\nabla^{E \otimes T^{*} M} \nabla^{E}\right)$ be the generalized Laplacian on $C^{\infty}(M, E)$ where $\nabla^{E}$ is a connection on $E$ and $\nabla^{T^{*} M \otimes E}$ the connection induced on $E \otimes T^{*} M$ by the connection $\nabla^{E}$ and the Levi-Civita connection on $M$.

Then $\sigma_{-n}\left(\left(\Delta^{E}\right)^{-m}\right)=|\xi|^{-n} I$ and

$$
\begin{aligned}
\operatorname{res}\left(\left(\Delta^{E}\right)^{-m}\right) & =\frac{1}{(2 \pi)^{n}} \int_{S^{*} M} \operatorname{tr}_{x}(I) d_{S}(\xi) \\
& =\frac{1}{(2 \pi)^{n}} \int_{S^{*} M} \operatorname{tr}_{x}(I) d_{S}(\xi) \\
& =\operatorname{rk}(E) \frac{\operatorname{vol}\left(S^{*} M\right)}{(2 \pi)^{n}} \\
& =\operatorname{rk}(E) \frac{\operatorname{vol}(M)}{2^{n-1}(m-1) ! \pi^{m}}
\end{aligned}
$$

whereas

$$
\operatorname{tr}_{0}^{\tau}\left(\left(\Delta^{E}\right)^{-m}\right)=0
$$

since

$$
\sigma_{-n}\left(\left(\Delta^{E}\right)^{-m}\right)=0
$$

The following theorem is the most important result of this chapter.

Theorem 4 All traces on the algebra $C \ell^{0}(M, E)$ are linear combinations the Wodzicki residue and leading symbol traces. They are continuous for the Fréchet topology of $C \ell^{0}(M, E)$.

Proof : The continuity property follows from that of the Wodzicki residue and the leading symbol traces discussed previously. But it can also be seen directly from the proof which involves topological isomorphisms; we write the 
proof in the algebraic setup since the corresponding topological result can be derived replacing ordinary tensor products $\otimes$ by their completion $\hat{\otimes}_{\pi}$.

We carry out the proof in the case $n>1$ which can easily be adapted to the case $n=1$.

By Lemma 3 the proof of Theorem 4 reduces to the computation of

$$
H H_{0}\left(C \ell^{0}(M) / C \ell^{-\infty}(M)\right)=H H_{0}\left(\mathcal{A}_{0}\right) \text {. }
$$

The Hochschild homology of $\mathcal{A}_{0}$ is computed by means of the spectral sequence ([We, M, CE] associated with its natural filtration (by orders). This spectral sequence was used in [W87] to compute the homology of differential operators and in $[\mathrm{BG}]$ to compute the homology of the algebra $\mathcal{A}=C \ell^{\mathbb{Z}}(M) / C \ell^{-\infty}(M)$. Because here the filtration is stopped at index 0 , the conclusion will slighty differ from $[\mathrm{BG}]$ and the proof requires extra care which is why we now review the main features of this algebraic machinery.

Let us consider the spectral sequence $\left(E_{p, q}^{r}, d^{r}\right)_{r \in \mathbb{N}}$, where $p \leq 0, q \geq 0$, associated with the natural filtration by orders on the Hochschild complex $C\left(\mathcal{A}_{0}\right)$. Provided this spectral sequence degenerates at a finite step $r$ and converges to $H H_{*}\left(\mathcal{A}_{0}\right)$, we can conclude that

$$
H H_{k}\left(\mathcal{A}_{0}\right) \simeq \oplus_{p+q=k} E_{p, q}^{r} .
$$

In particular, since we are looking for traces on $\mathcal{A}_{0}$, only diagonal terms $E_{-j, j}^{r}, j \geq$ 0 will be relevant for us.

The filtration (9) induces a grading by

$$
\mathcal{H}_{0}[p]:=F_{p}\left(\mathcal{H}_{0}\right) / F_{p-1}\left(\mathcal{H}_{0}\right)=h^{p} C^{\infty}\left(S^{*} M\right)
$$

which corresponds to formal power series with a single non vanishing term in degree $p$. Setting $k=p+q$ we have:

$$
\begin{aligned}
E_{p, q}^{0} & :=F_{p}\left(C_{p+q}\left(\mathcal{A}_{0}\right)\right) / F_{p-1}\left(C_{p+q}\left(\mathcal{A}_{0}\right)\right) \\
& \simeq \bigoplus_{\sum_{j=1}^{k} p_{j}=p} \mathcal{H}_{0}\left[p_{0}\right] \otimes \ldots \otimes \mathcal{H}_{0}\left[p_{k}\right] \\
& :=C_{k}\left(\mathcal{H}_{0}\right)[p],
\end{aligned}
$$

and $d^{0}: E_{p, q}^{0} \rightarrow E_{p, q-1}^{0}$ coincides under this isomorphism with the Hochschild boundary of $C_{*}\left(\mathcal{H}_{0}\right)$ restricted to the homogeneous part of degree $p$. Consequently, the complex $\left(E_{p, *}^{0}, d^{0}\right)$ is isomorphic to the complex $\left(C_{p+*}\left(\mathcal{H}_{0}\right)[p], b\right)$. Hence the terms $E_{*, *}^{1}$ (which are, by definition, homology groups of the complexes $\left.\left(E_{*, *}^{0}, d^{0}\right)\right)$ coincide under this isomorphism with the homogeneous part of degree $p$ of the Hochschild homology of $\mathcal{H}_{0}$ denoted by $H H_{*}\left(\mathcal{H}_{0}\right)[p]$. The isomorphism (6) now implies

$$
E_{p, q}^{1} \simeq H H_{k}\left(\mathcal{H}_{0}\right)[p] \simeq \Omega^{k}\left(\mathcal{H}_{0}\right)[p] .
$$

With these identifications, the boundary map $d^{1}: E_{p, q}^{1} \rightarrow E_{p-1, q}^{1}$ corresponds to $\delta: \Omega^{k}\left(\mathcal{H}_{0}\right)[p] \rightarrow \Omega^{k-1}\left(\mathcal{H}_{0}\right)[p-1]$ which was computed in [W87, BG]:

$$
\begin{aligned}
& \delta\left(f_{0} d f_{1} \wedge \ldots \wedge d f_{k}\right)=\sum_{i=1}^{k}(-1)^{i+1}\left\{f_{0}, f_{i}\right\} d f_{1} \wedge \ldots \hat{d f_{i}} \wedge \ldots \wedge d f_{k} \\
& \quad+\sum_{i<j}(-1)^{i+j} f_{0} d\left\{f_{i}, f_{j}\right\} \wedge d f_{1} \wedge \ldots \hat{d f_{i}} \wedge \ldots \wedge \hat{d f_{j}} \wedge \ldots \wedge d f_{k} .
\end{aligned}
$$


Let us now display the $E_{*, *}^{1}$ terms in the $(p, q)$-plane.

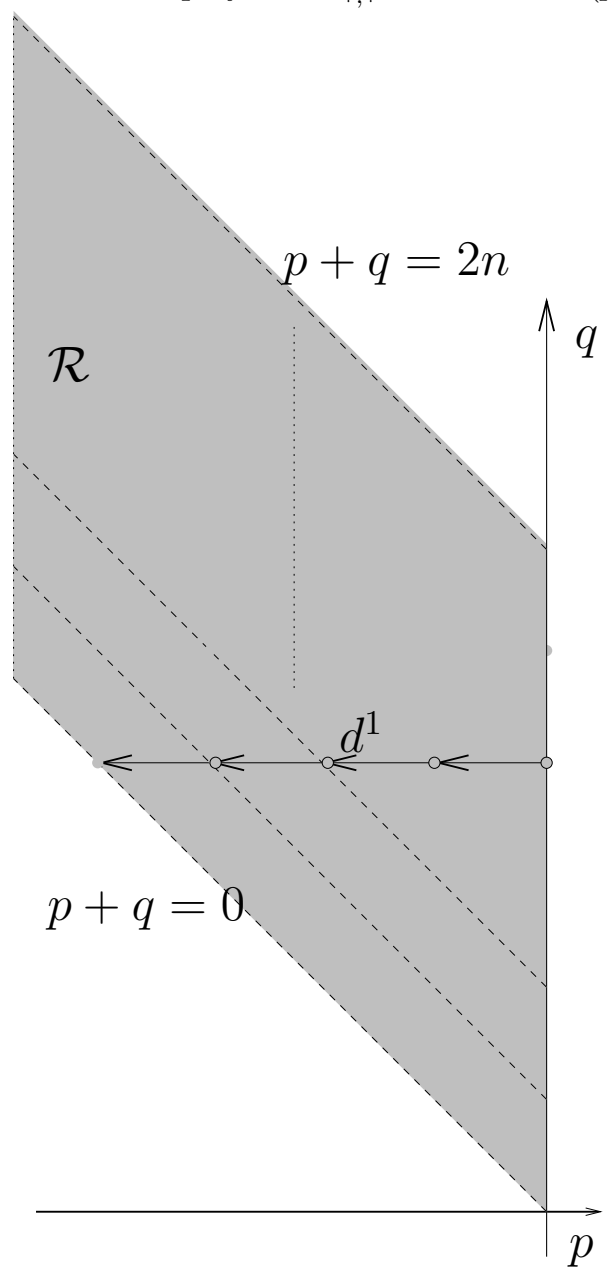

$E_{p, q}^{1}=0$ if $(p, q) \notin \mathcal{R}$. Next, from the fact that $\Omega^{k}(\mathcal{H})[p]=\Omega^{k}\left(\mathcal{H}_{0}\right)[p]$ for any negative $p$ it follows that:

$$
E_{p, k-p}^{2}= \begin{cases}\frac{\operatorname{ker} \delta: \Omega^{k}(\mathcal{H})[p] \rightarrow \Omega^{k-1}(\mathcal{H})[p-1]}{\operatorname{Im} \delta: \Omega^{k+1}(\mathcal{H})[p+1] \rightarrow \Omega^{k}(\mathcal{H})[p]} & \text { if } p<-1 \\ \frac{\operatorname{ker} \delta: \Omega^{k}(\mathcal{H})[-1] \rightarrow \Omega^{k-1}(\mathcal{H})[-2]}{\operatorname{Im} \delta: \Omega^{k+1}\left(\mathcal{H}_{0}\right)[0] \rightarrow \Omega^{k}(\mathcal{H})[-1]} & \text { if } p=-1 \\ \operatorname{ker} \delta: \Omega^{k}\left(\mathcal{H}_{0}\right)[0] \rightarrow \Omega^{k-1}(\mathcal{H})[-1] & \text { if } p=0\end{cases}
$$

Correspondingly, we consider three cases $p<-1, p=-1$ and $p=0$.

To compute $E_{p, q}^{2}$ with $p<-1$, we use [B]. Let $\omega$ be the canonical symplectic form of $T^{*} M$ and $G$ be the two contravariant tensor field on $T^{*} M$ defined locally by $G=\sum_{i} \partial / \partial x_{i} \wedge \partial / \partial \xi_{i}$. The $*$ operator is defined by

$$
\begin{aligned}
\Omega^{k}\left(T^{*} M\right) & \rightarrow \Omega^{2 n-k}\left(T^{*} M\right) \\
\alpha & \mapsto * \alpha \quad \text { with } \beta \wedge * \alpha=\Lambda^{k} G(\beta, \alpha) \cdot \omega^{n} / n ! \quad \forall \beta \in \Omega^{k}\left(T^{*} M\right),
\end{aligned}
$$


We refer to $[\mathrm{B}]$ for a complete presentation. The reason to introduce this $*$ operator is the following commutative diagram :

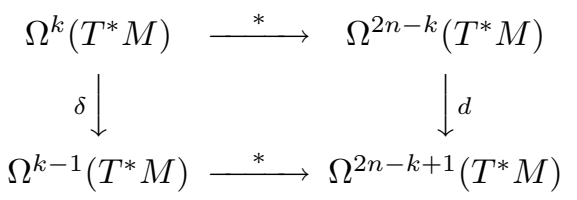

Moreover, the map $*$ gives isomorphisms $\Omega^{k}(\mathcal{H})[m] \simeq \Omega^{2 n-k}(\mathcal{H})[n+m-k]$ for we have

$$
*\left(d x^{I} \wedge d \xi^{J}\right)= \pm d x^{\bar{J}} \wedge d \xi^{\bar{I}} .
$$

Since $\Omega^{k}(\mathcal{H})[m] \simeq \Omega^{k}\left(\mathcal{H}_{0}\right)[m]$ for all $m<-1$, we get the following commutative diagram for $p<-1$ :

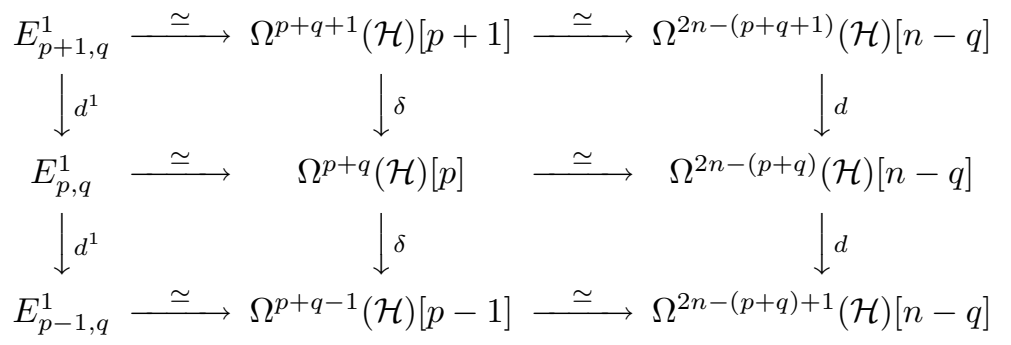

To deduce the value of $E_{p, q}^{2}$ from (13) in the cases $p<-1$, it suffices to compute the cohomology of the complexes $\left(\Omega^{*}(\mathcal{H})[m], d\right)$ for $m \in \mathbb{Z}$.

For $m \neq 0$, let us define the operator

$$
K_{m}: \Omega^{*}(\mathcal{H})[m] \rightarrow \Omega^{*-1}(\mathcal{H})[m]
$$

by :

$$
\omega=h^{m}\left(\alpha+\frac{d h}{h} \wedge \beta\right) \in \Omega^{k}(\mathcal{H})[m] ; \quad K_{m} \omega=\frac{h^{m}}{m} \beta .
$$

A straightforward computation yields :

$$
d K_{m} \omega+K_{m} d \omega=\omega .
$$

Thus, whenever $m \neq 0$, the complex $\left(\Omega^{*}(\mathcal{H})[m], d\right)$ has trivial cohomology.

If $m=0$, then

$$
\Omega^{k}(\mathcal{H})[0]=\Omega^{k}\left(S^{*} M\right) \oplus \frac{d h}{h} \wedge \Omega^{k-1}\left(S^{*} M\right)
$$

and the map :

$$
\begin{array}{ccc}
H_{d R}^{k}\left(S^{*} M\right) \oplus H_{d R}^{k-1}\left(S^{*} M\right) & \longrightarrow & H^{k}\left(\Omega^{*}(\mathcal{H})[0]\right) \\
{[\alpha]+[\beta]} & \mapsto & {\left[\alpha+\frac{d h}{h} \wedge \beta\right]}
\end{array}
$$

is an isomorphism, which is easy to check.

This ends the computations of $E^{2}$-terms with $p<-1$ for which we get :

For all $p<-1, \quad E_{p, q}^{2} \simeq \begin{cases}0 & \text { if } q \neq n \\ H_{d R}^{n-p}\left(S^{*} M\right) \oplus H_{d R}^{n-p-1}\left(S^{*} M\right) & \text { if } q=n\end{cases}$ 
Let us consider the case $p=-1$. Looking again at (12), we see that the term $E_{-1, q}^{2}$ can not be computed in the same way as above. Indeed, $\Omega^{k}\left(\mathcal{H}_{0}\right)[0] \neq$ $\Omega^{k}(\mathcal{H})[0]$, whence the diagram (13) should be modified and one expects the identification of the $E^{2}$-terms with de Rham cohomology groups to be less straight forward.

In fact, since only diagonal terms in the $E^{2}$-plane will contribute to $H H_{0}\left(\mathcal{A}_{0}\right)$, one only needs to evaluate $E_{-1,1}^{2}$. We have :

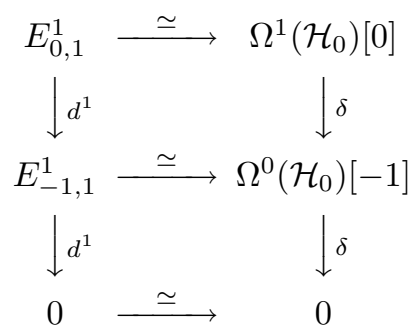

Let us consider the canonical one form $\alpha$ whose differential gives the symplectic form on $T^{*} M$ and let $f \in \Omega^{0}\left(\mathcal{H}_{0}\right)[-1]$. We claim that :

$$
\delta(f \alpha)=(n-1) f .
$$

Indeed, in local coordinates $\alpha=-\xi_{i} d x^{i}$ and :

$$
\begin{aligned}
\delta(f \alpha) & =-\delta\left(f \xi_{i} d x^{i}\right)=-\left\{f \xi_{i}, x^{i}\right\} \\
& =\partial_{\xi_{j}}\left(\xi_{i} f\right) \partial_{x^{j}}\left(x^{i}\right)-\partial_{x^{j}}\left(\xi_{i} f\right) \partial_{\xi_{j}}\left(x^{i}\right) \\
& =\partial_{\xi_{i}}\left(\xi_{i} f\right)=n f+\xi_{i} \partial_{\xi_{i}}(f) \\
& =n f-f .
\end{aligned}
$$

The last equality holds since $f$ is an homogeneous function of order -1 .

Moreover $f \alpha$ belongs to $\Omega^{1}\left(\mathcal{H}_{0}\right)[0]$ : indeed, using a partition of unity, one can write $f \alpha$ as a sum of forms $f_{0} d f_{1}$ where $f_{0}, f_{1}$ are homogeneous of degree 0 . Therefore :

$$
E_{-1,1}^{2}=0 \text { if } n>1 \text {. }
$$

Finally, for $p=0$, we already know that :

$$
E_{0, q}^{2} \simeq \operatorname{ker}\left(\delta: \Omega^{q}\left(\mathcal{H}_{0}\right)[0] \rightarrow \Omega^{q-1}\left(\mathcal{H}_{0}\right)[-1]\right)
$$

may be non vanishing for $0 \leq q \leq 2 n$ and for the term which interest us most we have:

$$
E_{0,0}^{2} \simeq \Omega^{0}\left(\mathcal{H}_{0}\right)[0]=C^{\infty}\left(S^{*} M\right)
$$

It follows from the finiteness of the number of non vanishing $E_{p, q}^{2}$ that the spectral sequence must degenerate, and looking at the location of non trivial $E^{2}$ terms, we see that this degeneracy holds at most at the $(n+1)$-th step. 


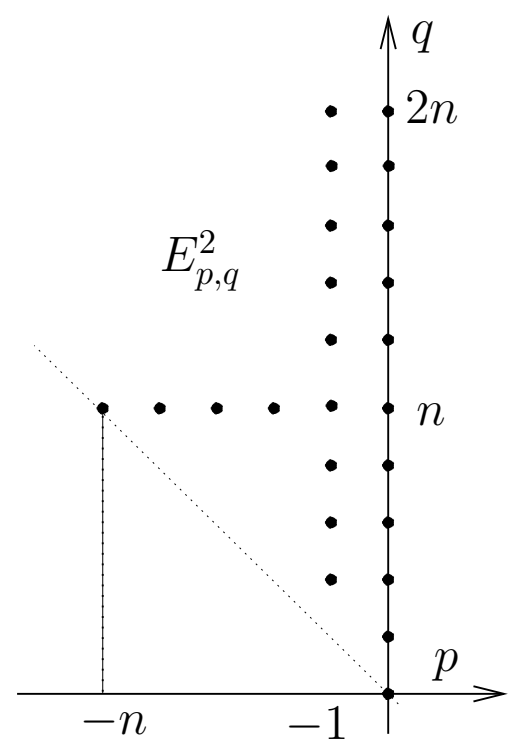

This together with lemma (5) shows that assumptions of ([We], theorem 5.5.10) are fulfilled, hence the spectral sequence converges to $H H_{*}(\mathcal{A})$.

Since we are looking for traces, we are only interested in :

$$
H H_{0}(\mathcal{A}) \simeq E_{-n, n}^{n+1} \oplus E_{0,0}^{n+1} .
$$

Let us prove that $E_{-n, n}^{n+1}=E_{-n, n}^{2}$ and that $E_{0,0}^{n+1}=E_{0,0}^{2}$. Note that if $n>1$

$$
\begin{array}{rlc}
E_{-n, n}^{2} \oplus E_{0,0}^{2} & \simeq & H_{d R}^{2 n}\left(S^{*} M \times S^{1}\right) \oplus \Omega^{0}\left(\mathcal{H}_{0}\right)[0] \\
& \simeq & \mathbb{C} \oplus C^{\infty}\left(S^{*} M\right)
\end{array}
$$

and carefully looking at the previous identifications, one sees that the projection map

$$
C_{0}(\mathcal{A}) \longrightarrow E_{-n, n}^{2} \oplus E_{0,0}^{2}
$$

is given by :

$$
a=\sum a_{j} \longmapsto\left(\int_{S^{*} M} a_{-n}(v) d v, a_{0}(v)\right),
$$

where $d v=d x d_{x} \xi$ is the volume form of $S^{*} M$.

Since the differential maps $d^{r}$ have bidegree $(-r, r-1)$, they must vanish on $E_{0,0}^{r}$ for all $r \geq 2$ (the target space is always trivial). Moreover, there is no non trivial $E_{p, q}^{r}$ mapped to $E_{0,0}^{r}$ by $d^{r}$. Hence :

$$
E_{0,0}^{n+1}=E_{0,0}^{2}
$$

There is no non trivial target for $d^{r}$ acting on $E_{-n, n}^{r}$ but $E_{-n, n}^{r}$ can be reached by :

$$
d^{n-1}: E_{-1,2}^{n-1} \rightarrow E_{-n, n}^{n-1}
$$

and next by :

$$
d^{n}: E_{0,1}^{n} \rightarrow E_{-n, n}^{n}
$$


Since $E_{-n, n}^{n-1}=E_{-n, n}^{2}$ is a one dimensional vector space over $\mathbb{C}$, either (17) vanishes or it is onto, and in the first case, the same argument applies for (18).

The non vanishing of any of these two differential maps would imply that $E_{-n, n}^{n+1}=0$ and hence that the Wodzicki residue vanishes on zero order symbols, which is obviously wrong. We can therefore conclude that $E_{-n, n}^{n+1}=E_{-n, n}^{2}$ and that :

$$
H H_{0}(\mathcal{A}) \simeq E_{-n, n}^{2} \oplus E_{0,0}^{2} \simeq \mathbb{C} \oplus C^{\infty}\left(S^{*} M\right) .
$$

with isomorphism induced by (16). Hence traces on the algebra of non positive integer order classical pseudodifferential operators are linear combination of the Wodzicki residue and of leading symbols traces given by any current :

$$
\tau \in\left(C^{\infty}\left(S^{*} M\right)\right)^{\prime} ; \quad \operatorname{tr}_{\tau}: a \mapsto \tau\left(a_{0}\right)
$$

as claimed in the statement of the theorem.

\section{Determinants on zero order invertible pseu- dodifferential operators}

\subsection{The fundamental group of the class of zero order in- vertible pseudodifferential operators}

Wodzicki showed that the spectral asymmetry of elliptic classical pseudodifferential operators is encoded by pseudodifferential operators of the form:

$$
\Pi_{\theta, \theta^{\prime}}(Q):=\frac{1}{2 i \pi} \int_{C_{\theta, \theta^{\prime}, r}} \lambda^{-1} Q(Q-\lambda)^{-1} d \lambda
$$

where

$$
C_{\theta, \theta^{\prime}, r}:=\left\{\rho e^{i \theta}, \infty>\rho \geq r\right\} \cup\left\{r e^{i t}, \theta \leq t \leq \theta^{\prime}\right\} \cup\left\{\rho e^{i \theta^{\prime}}, r \leq \rho<\infty\right\},
$$

with $Q \in C \ell(M, E)$ elliptic with positive order and whereby $r$ is chosen small enough so that non non-zero eigenvalue of $Q$ lies in the disc $|\lambda| \leq r$. It turns out that $\Pi_{\theta, \theta^{\prime}}(Q)$ is a projection on $L^{2}(M, E)$ (see $[\mathrm{Po}]$ ) and either a zero'th order pseudodifferential operator or a smoothing operator. For $Q$ of order $m$ with leading symbol $q_{m}$, its leading symbol reads:

$$
\Pi_{\theta, \theta^{\prime}}\left(q_{m}\right):=\frac{1}{2 i \pi} \int_{C_{\theta, \theta^{\prime}, r}} \lambda^{-1} q_{m}\left(q_{m}-\lambda\right)^{-1} d \lambda .
$$

The following proposition shows that these pseudodifferential projectors generate the fundamental group $\pi_{1}\left(G L_{\infty}\left(C \ell^{0, *}(M)\right)\right)$.

\section{Proposition 5}

$\pi_{1}\left(G L_{\infty}\left(C \ell^{0}(M)\right)\right)$ is generated by loops $e^{2 i \pi t \Pi_{\theta, \theta^{\prime}}(Q)}$ where $Q \in C \ell(M, E)$ is any elliptic operator with positive order and $E$ any vector bundle of finite rank over $M$. 
Remark 7 Let

$$
C \ell_{I d}^{0, *}(M, E):=\left\{A \in C \ell^{0, *}(M, E), \quad \sigma_{L}(A)=I d\right\} .
$$

It was shown in [BW] (Proposition 15.4) that

$$
\begin{aligned}
\pi_{k}\left(C \ell_{I d}^{0, *}(M, E)\right) & \simeq 0 \text { for } k \text { even } \\
& \simeq \mathbb{Z} \text { for } k \text { odd }
\end{aligned}
$$

To do so, the authors compare the homotopy groups of $C \ell_{I d}^{0, *}(M, E)$ with the known (via Bott periodicity) homotopy groups of

$$
G L_{\text {res }}\left(L^{2}(M, E)\right):=\left\{A \in G L\left(L^{2}(M, E)\right), \quad A-1 \text { has finite rank }\right\} .
$$

\section{Proof of the proposition:}

For any algebra $\mathcal{A}$, let $K_{0}(\mathcal{A})$ denote the group of formal differences of homotopy classes of idempotents in $g l_{\infty}(A)$. When $\mathcal{A}$ is a good topological algebra $([\mathrm{Bo}])$, the Bott periodicity isomorphism:

$$
\begin{array}{ccc}
K_{0}(\mathcal{A}) & \longrightarrow & \pi_{1}\left(G L_{\infty}(\mathcal{A})\right) \\
{[P]} & \longmapsto & e^{2 i \pi t P}
\end{array}
$$

holds. Since for any vector bundle $E$ over $M$, the algebra $C l^{0}(M, E)$ is a good topological algebra (which essentially boils down to the fact that the inverse of a classical pseudodifferential operator remains a classical pseudodifferential operator), applying (19) to $\mathcal{A}=C l^{0}(M, E)$ reduces the proof down to checking that $K_{0}\left(C l^{0}(M, E)\right)$ is generated by idempotents $\Pi_{\theta, \theta^{\prime}}(Q)$. Recall the exact sequence :

$$
0 \longrightarrow C l^{-1}(M, E) \longrightarrow C l^{0}(M, E) \stackrel{\sigma^{L}}{\longrightarrow} C^{\infty}\left(S^{*} M, p^{*}(\operatorname{End} E)\right) \longrightarrow 0
$$

where $\sigma_{L}: A \mapsto \sigma^{L}(A)$ denotes the leading symbol map on classical pseudodifferential operators, which gives rise to a long exact sequence in $K$-theory [WO]:

$$
\begin{aligned}
& \begin{array}{c}
K_{0}\left(C l^{-1}(M, E)\right) \\
\uparrow \text { Ind }
\end{array} \quad \rightarrow \quad K_{0}\left(C l^{0}(M, E)\right) \stackrel{\sigma_{0}^{L}}{\rightarrow} \quad K_{0}\left(C^{\infty}\left(S^{*} M, p^{*}(\operatorname{End} E)\right)\right) \\
& K_{1}\left(C^{\infty}\left(S^{*} M, p^{*}(\operatorname{End} E)\right)\right) \stackrel{\sigma_{1}^{L}}{\leftarrow} K_{1}\left(C l^{0}(M, E)\right) \leftarrow \quad K_{1}\left(C l^{-1}(M, E)\right)=0
\end{aligned}
$$

Now on the grounds of results by Wodzicki, Ponge proves in $[\mathrm{Po}]$ that $K_{0}\left(C^{\infty}\left(S^{*} M, p^{*}(\operatorname{End} E)\right)\right)$ is generated by the classes $\Pi_{\theta, \theta^{\prime}}\left(\sigma_{m}(Q)\right)$ where $\sigma_{m}(Q)$ is the principal symbol of an elliptic operator $Q \in C l(M, E)$ with positive order $m$. This result combined with the obvious surjectivity of the map $\sigma_{0}^{L}$ in the diagram (20) yields the result.

\subsection{A uniqueness result for multiplicative determinants on zero order operators}

In order to apply the results of section 1 to the traces res and $\operatorname{tr}_{0}^{\tau}$ on $C \ell^{0}(M, E)$ so as to build determinants on the Lie group $\mathcal{G}=C \ell^{0, *}(M, E)$, we need the following preliminary result. 
Lemma 6 For $\lambda=$ res or $\lambda=\operatorname{tr}_{0}^{\tau}$ with $\tau \in C^{\infty}\left(S^{*} M \text {, End }\left(p^{*} E\right)\right)^{\prime}$, the image of $\pi_{1}\left(C \ell_{0}^{*}(M, E)\right)$ under the map

$$
\begin{aligned}
\operatorname{Det}_{\lambda}: \mathcal{P} C \ell^{0, *}(M, E) & \rightarrow \mathbb{C} \\
\gamma & \mapsto e^{\int_{0}^{1} \lambda\left(\dot{\gamma} \gamma^{-1}\right)}
\end{aligned}
$$

is trivial.

Proof: As in [KV2] section 4, we first reduce the problem to the case of a trivial bundle $1_{N}$. There is a smooth vector bundle $F$ on $M$ and an integer $N$ such that $E \oplus F \simeq 1_{N}$ where $1_{N}$ is the trivial rank $N$ vector bundle on $M$. Extending $\lambda$ by 0 , we have $e^{\int_{0}^{1} \lambda\left(\dot{\gamma} \gamma^{-1}\right)}=e^{\int_{0}^{1} \lambda\left(\dot{\tilde{\gamma}} \tilde{\gamma}^{-1}\right)}$ with $\tilde{\gamma}=\gamma \oplus I d_{F}$ and hence

$$
\operatorname{Det}_{\lambda}\left(\pi_{1}\left(C l^{0, *}(M, E)\right)\right)=\operatorname{Det}_{\lambda}\left(\pi_{1}\left(C l^{0, *}\left(M, 1_{N}\right)\right)\right) .
$$

Since this holds independently of the choice of $N$, we get:

$$
\operatorname{Det}_{\lambda}\left(\pi_{1}\left(C l^{0, *}(M, E)\right)\right)=\operatorname{Det}_{\lambda}\left(\pi_{1}\left(G L_{\infty}\left(C l^{0, *}(M)\right)\right) .\right.
$$

By Proposition 3.1, we are therefore left to evaluate the leading symbol trace $\operatorname{tr}_{0}^{\tau}$ and the Wodzicki residue trace on pseudodifferential projectors $\Pi_{\theta, \theta^{\prime}}(Q)$ where $Q \in C l\left(M, 1_{N}\right)$ is elliptic with positive order. By equation (10) we know that $\operatorname{tr}_{0}^{\tau}$ takes integer values on pseudodifferential idempotents so that $\operatorname{det}_{0}^{\tau}\left(e^{2 i \pi t \pi_{\theta, \theta^{\prime}}(Q)}\right)=1$.

On the other hand it was proved by Wodzicki [W] (see also [Po]) that pseudodifferential projectors have vanishing Wodzicki residue so that for $\lambda=$ res we also have $\operatorname{Det}_{\lambda}\left(e^{2 i \pi t \pi_{\theta, \theta^{\prime}}(Q)}\right)=1$.

Recall from Definition 2 that a determinant map on a Lie group $\mathcal{G}$ is a multiplicative map Det $: \tilde{\mathcal{G}} \rightarrow \mathbb{C}^{*}$ on its pathwise component of the identity.

Theorem 5 Determinant maps of class $C^{1}$ on $C \ell^{0, *}(M, E)$ are given by a two parameter family

$$
\operatorname{Det}_{a, b}:=e^{a \int_{0}^{1} \operatorname{res}\left(\dot{\gamma}_{A} \gamma_{A}^{-1}\right)+b \int_{0}^{1} \operatorname{tr}_{0}^{\tau}\left(\dot{\gamma}_{A} \gamma_{A}^{-1}\right)}, \quad a, b \in \mathbb{C}, \tau \in C^{\infty}\left(S^{*} M\right)^{\prime}
$$

where:

- $\operatorname{det}_{\mathrm{res}}:=\operatorname{Det}_{1,0}$ is the Wodzicki multiplicative residue (also called the "exotic (logarithmic) determinant" [W], $[K])$ :

$$
\begin{aligned}
\tilde{C} \ell^{0, *}(M, E) & \rightarrow \mathbb{C}^{*} \\
A & \mapsto \operatorname{det}_{\mathrm{res}}(A):=e^{\int_{0}^{1} \operatorname{res}\left(\dot{\gamma}_{A} \gamma_{A}^{-1}\right)},
\end{aligned}
$$

- and $\operatorname{det}_{0}^{\tau}:=\operatorname{Det}_{0,1}$ are leading symbol determinants [PR1]:

$$
\begin{aligned}
\tilde{C} \ell^{0, *}(M, E) & \rightarrow \mathbb{C}^{*} \\
A & \mapsto \operatorname{det}_{0}^{\tau}(A):=e^{\int_{0}^{1} \operatorname{tr}_{0}^{\tau}\left(\dot{\gamma}_{A} \gamma_{A}^{-1}\right)} .
\end{aligned}
$$

Here $\gamma_{A} \in \mathcal{P} C \ell^{0, *}(M, E)$ is a path ending at $A$ and $\tau$ is a current in $C^{\infty}\left(S^{*} M\right)^{\prime}$. 
They make the following diagram commute

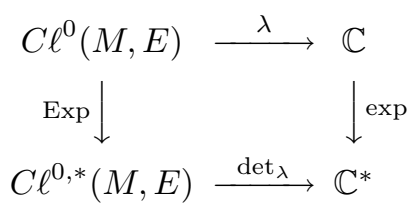

where $\lambda$ and $\operatorname{det}_{\lambda}$ stand for res and $\operatorname{det}_{\text {res }}$, respectively for $\operatorname{tr}_{0}^{\tau}$ and $\operatorname{det}_{0}^{\tau}$. Here Exp is the exponential map on $C \ell^{0, *}(M, E)$ whereas $\exp$ is the ordinary exponential on complex numbers.

Remark 8 Recall that for the $\zeta$-determinant

$$
\operatorname{det}_{\zeta}(A)=e^{-\zeta_{A}^{\prime}(0)}
$$

defined in terms of the $\zeta$-function $\zeta_{A}$ of $A$, we have

$$
\operatorname{det}_{\zeta}(\lambda A)=\lambda^{\zeta_{A}(0)} \operatorname{det}_{\zeta}(A) \quad \forall \lambda \in \mathbb{R}^{+}
$$

so that $\zeta_{A}(0)$ plays the role of the dimension $\operatorname{det}(\lambda A)=\lambda^{n} \operatorname{det}(A)$ for the ordinary determinant det on $G L_{n}(\mathbb{C})$. For the residue determinant we have

$$
\operatorname{det}_{\text {res }}(\lambda A)=\operatorname{det}_{\text {res }}(A) \quad \forall \lambda \in \mathbb{R}^{+}
$$

and for the leading symbol determinant

$$
\operatorname{det}_{0}^{\tau}(\lambda A)=\lambda^{\mathrm{rkE}} \operatorname{det}_{0}^{\tau}(A) \quad \forall \lambda \in \mathbb{R}^{+}
$$

with $\mathrm{rkE}$ the rank of the vector bundle $E$.

Let us illustrate the determinants introduced in this theorem by an example taken from [Sc]. It relies on the following observation

Lemma 7 Let $A \in C \ell^{0, *}(M, E)$ an admissible elliptic operator of negative order. Then $\operatorname{tr}_{0}^{\tau}(\log (1+A))=0$ and

$$
\operatorname{res}(\log (1+A))=\sum_{k=1}^{\infty}(-1)^{k+1} \frac{\operatorname{res}\left(A^{k}\right)}{k},
$$

where the sum is in fact a finite sum.

Proof: Since $A$ has negative order, we have the following equivalence on the symbol level

$$
\sigma_{\log (1+A)} \sim \sum_{k=1}^{\infty}(-1)^{k+1} \frac{\sigma_{A^{k}}}{k} .
$$

Since the zero-th order part vanishes, $\operatorname{tr}_{0}^{\tau}(\log (1+A))=0$. Similarly, the formula for the Wodzicki residue follows from integrating the (positively) homogeneous part of order $-n$ over the cotangent sphere. Only a finite number of terms remain since the order of $A^{k}$ decreases and the Wodzicki residue vanishes on operators of order $<-n$. 
Example 3 Let $M$ be an even $n=2 k$-dimensional closed Riemannian manifold and let as before $E$ be a vector bundle over $M$. The operator $1+\left(\Delta^{E}\right)^{-k} \in$ $C \ell^{0, *}(M, E)$ has a well defined logarithm and by Lemma 7 we get

$$
\begin{aligned}
\operatorname{det}_{\mathrm{res}}\left(1+\left(\Delta^{E}\right)^{-k}\right) & =e^{\operatorname{res}\left(\Delta^{E}\right)^{-k}} \\
& =\exp \left(\frac{\operatorname{vol}\left(S^{*} M\right)}{2^{2 k-1}(k-1) ! \pi^{k}}\right)
\end{aligned}
$$

whereas

$$
\begin{aligned}
\operatorname{det}_{0}^{\tau}\left(1+\left(\Delta^{E}\right)^{-k}\right) & =e^{\operatorname{tr}_{0}^{\tau} \log 1+\left(\Delta^{E}\right)^{-k}} \\
& =e^{\operatorname{tr}_{0}^{\tau}\left(\Delta^{E}\right)^{-k}} \\
& =1
\end{aligned}
$$

Proof: First of all, $\operatorname{det}_{\text {res }}$ and $\operatorname{det}_{0}^{\tau}$ are well defined as a result of Lemma 6 and Proposition 2. Since res and $\operatorname{tr}_{0}^{\tau}$ are Ad-invariant and continuous, it follows from Proposition 2 that $\operatorname{det}_{\text {res }}$ and $\operatorname{det}_{0}^{\tau}$ are multiplicative and of class $C^{1}$. By Proposition 1 , any other determinant map $\Lambda_{0}: \tilde{C} \ell^{0, *}(M, E) \rightarrow \mathbb{C}^{*}$ which is of class $C^{1}$ induces an Ad-invariant continuous linear map $\lambda: C \ell^{0}(M, E) \rightarrow \mathbb{C}$. The result then follows from the fact that by Theorem 4, res and $\operatorname{tr}_{0}^{\tau}$ are (up to a multiplicative factor which gives rise to powers of the determinants) the only such maps on $C \ell^{0}(M, E)$. The commutativity of the diagram follows from Theorem 3.

Let $C \ell_{a d m}^{0, *}(M, E)$ denote the class of operators $A \in C \ell^{0, *}(M, E)$ which admit a spectral cut $\theta$ and let us as before denote by $\log _{\theta} A$ the logarithm of an operator $A$ in that class.

\section{Proposition 6}

$$
C \ell_{a d m}^{0, *}(M, E) \subset \tilde{C} \ell^{0, *}(M, E)
$$

and

$$
\operatorname{det}_{\text {res }}(A)=e^{\operatorname{res}\left(\log _{\theta}(A)\right)} ; \quad \operatorname{det}_{0}^{\tau}(A)=e^{\operatorname{tr}_{0}^{\tau}\left(\log _{\theta}(A)\right)} \quad \forall A \in C \ell_{a d m}^{0, *}(M, E)
$$

independently of the choice of spectral cut $\theta$.

\section{Proof:}

- Since $A$ has a spectral cut $\theta, \gamma_{A}(t):=A_{\theta}^{t}$ which has constant order 0 since $A$ is invertible with order 0 , defines a path in $\mathcal{P} C \ell^{0, *}(M, E)$ which links $A$ to 1 and proves the first part of the statement.

- We give two proofs of the second part of the proposition. The first one follows directly from the fact that $A=\operatorname{Exp}\left(\log _{\theta} A\right.$ ) (independently of the choice of spectral cut) and the relation $\operatorname{det}_{\lambda}(\operatorname{Exp}(B))=\exp (\lambda(B))$ resulting from the commutativity of the diagram in Theorem 5 .

- For the sake of completeness, we give an alternative proof by hand. Let us check that $\Lambda_{0}$ is differentiable at identity and that $D_{1} \Lambda_{0}=\lambda$. Since 
$d(A-\mu)^{-1}=-(A-\mu)^{-1} d A(A-\mu)^{-1}$, using the Ad-invariance of $\lambda$ combined with an integration by parts formula, we have

$$
\begin{aligned}
d\left(\lambda\left(\log _{\theta}\left(\gamma_{A}\right)\right)\right) & =\frac{i}{2 \pi} d \int_{\Gamma_{\theta}} \lambda\left(\log _{\theta} \mu(A-\mu)^{-1}\right) d \mu \\
& =\frac{i}{2 \pi} \int_{\Gamma_{\theta}} \log _{\theta} \mu \cdot \lambda\left(d(A-\mu)^{-1}\right) d \mu \\
& =-\frac{i}{2 \pi} \int_{\Gamma_{\theta}} \log _{\theta} \mu \cdot \lambda\left((A-\mu)^{-1} d A(A-\mu)^{-1}\right) d \mu \\
& =-\frac{i}{2 \pi} \int_{\Gamma_{\theta}} \log _{\theta} \mu \cdot \lambda\left(d A(A-\mu)^{-2}\right) d \mu \\
& =\frac{i}{2 \pi} \lambda\left(d A \int_{\Gamma_{\theta}} d \mu \mu^{-1}(A-\mu)^{-1}\right) \\
& =\lambda\left(d A A^{-1}\right)
\end{aligned}
$$

so that $D_{1} \Lambda_{0}=\lambda$.

Hence

$$
\begin{aligned}
\Lambda_{0}(A) & =e^{\lambda\left(\log _{\theta}\left(\gamma_{A}\right)\right)} \\
& =e^{\int_{0}^{1} \frac{d}{d t}\left(\lambda\left(\log _{\theta} \gamma_{A}\right)\right)} \\
& =e^{\left.\int_{0}^{1} \lambda\left(\gamma_{A}^{-1} \dot{\gamma}_{A}\right)\right)} \\
& =\operatorname{det}_{\lambda}(A)
\end{aligned}
$$

where $\gamma_{A} \in \mathcal{P} C \ell(M, E)$ ends at $A$.

Remark 9 Another choice of spectral cut leads to another choice of path $\gamma_{A}$ but we saw in Proposition 2 that $\operatorname{det}_{\lambda}(g)$ is independent of the choice of path $\gamma \in \mathcal{P G}$ with $\gamma(1)=g$.

\section{Multiplicative determinants on invertible clas- sical pseudodifferential operators of all orders}

\subsection{Logarithmic operators}

We extend the class $C \ell^{0}(M, E)$ in order to allow for logarithms of elliptic operators.

Let as in section $2, U$ be an open subset in $\mathbb{R}^{n}$ and let $W$ be a euclidean vector space. We set for any $m \in \mathbb{R}$

$$
C S^{m, 1}(U, W):=\left\{\sigma(x, \xi)=\sigma^{0}(x, \xi)+\sigma^{1}(x, \xi) \log |\xi|, \quad \sigma_{0}, \sigma_{1} \in C S^{m}(U, W)\right\}
$$

which correspond to 1-logpolyhomogeneous operators in the sense of [Le]. Let us consider the following subsets of $C S^{0,1}(U, W)$ :

$$
C S_{\log , q}(U, W):=\left\{\sigma(x, \xi)=\sigma^{0}(x, \xi)+q \log |\xi|, \quad \sigma_{0} \in C S^{0}(U, W)\right\}
$$


and

$$
C S_{\log }(U, W):=\bigcup_{q \in \mathbb{R}} C S_{\log , q}(U, W) .
$$

Given two local trivialisations $E_{\left.\right|_{U}} \simeq U \times W, E_{\left.\right|_{V}} \simeq V \times W$ over two open subsets $U, V$ in $M$ of some vector bundle $E$ over $M$ modelled on $W$, a pseudodifferential operator acting on sections of $E$ whose symbol lies in $C S^{m, 1}(U \cap V, W)$ (resp. in $C S_{\log , q}(U \cap V, W)$ ) in the first local trivialisation over $U \cap V$ also lies in $C S^{m, 1}(U \cap V, W)$ (resp. in $C S_{\log , q}(U \cap V, W)$ ) in the second local trivialisation so that it makes sense to define the linear spaces $C \ell^{m, 1}(M, E)$ (resp. $\left.C \ell_{\log , q}(M, E)\right)$ of 1-log-polyhomogeneous (resp. of logarithmic operators) operators pseudodifferential operators acting on smooth sections of $\mathrm{E}$, whose symbol lies in $C S^{m, 1}(U, W)\left(\right.$ resp. $\left.C \ell_{\log }(M, E)\right)$ in some local trivialisation over $U$.

Remark $10 C \ell_{\log , 0}(M, E)=C \ell^{0}(M, E)$.

Recall from [Se] that an operator $Q \in C \ell^{*}(M, E)$ with spectral cut $\theta$ has complex powers $Q_{\theta}^{z}=\frac{i}{2 \pi} \int_{\Gamma_{\theta}} \lambda^{z}(Q-\lambda)^{-1} d \lambda$ where $\Gamma_{\theta}$ is a contour around the spectrum and a corresponding logarithm

$$
\log _{\theta} Q:=\left.\frac{d}{d z}\right|_{z=0} Q_{\theta}^{z}
$$

Lemma 8 The logarithm $\log _{\theta} Q$ of an operator $Q \in C \ell^{*}(M, E)$ of order $q$ with spectral cut $\theta$ lies in $C \ell_{\log , q}(M, E)$ which reads

$$
C \ell_{\log , q}(M, E)=C \ell^{0}(M, E)+\log _{\theta} Q
$$

independently of the choice of $Q$ of order $q$. It is a Fréchet affine space with associated linear space $C \ell^{0}(M, E)$ and is stable under the adjoint action of $C \ell^{*}(M, E)$.

Any operator $A \in C \ell_{\log , q}(M, E)$ of order a can be written in a unique way as

$$
A=\frac{a}{q} \log _{\theta} Q+A^{0}, \quad A^{0} \in C \ell^{0}(M, E)
$$

and

$$
C \ell_{\log }(M, E)=C \ell_{0}(M, E) \oplus \mathbb{R} \log _{\theta} Q
$$

is a Fréchet Lie algebra $C \ell_{0}(M, E)$ for the bracket $\widetilde{[\cdot, \cdot]}$ defined by:

$$
\left[\alpha \log _{\theta} Q+\widetilde{A^{0}, \beta} \log _{\theta} Q+B^{0}\right]:=\alpha\left[\log _{\theta} Q, B^{0}\right]-\beta\left[\log _{\theta} Q, A^{0}\right]+\left[A^{0}, B^{0}\right] .
$$

Remark 11 The algebra $C \ell_{\log }(M, E)$ arises in the context of pseudodifferential operators on manifolds with boundaries in [MN] and in the context of $\zeta$ determinants in [O, KV1, KV2].

Proof: The inclusion $C \ell_{0}(M, E)+\log _{\theta} Q \subset C \ell_{\log , q}(M, E)$ follows from the fact that in a local trivialisation $E_{\left.\right|_{U}} \simeq U \times W$ over $U, \log _{\theta} Q$ has a symbol $\sigma_{\log _{\theta} Q}$ with $\sigma_{\log _{\theta} Q}-q \log |\xi| \in C S^{0}(U, W)$. Similarly, the converse inclusion $C \ell_{\log , q}(M, E) \subset C \ell^{0}(M, E)+\log _{\theta} Q$ follows from the fact that if $\sigma_{A}-q \log |\xi| \in$ $C S^{0}(U, W)$ then $\sigma_{A}-\sigma_{\log _{\theta} Q} \in C S^{0}(U, W)$ and hence $A-\log _{\theta} Q \in C \ell^{0}(M, E)$. The Fréchet space structure on $C \ell^{0}(M, E)$ induces a natural Fréchet affine space 
structure on $C \ell_{\log , q}(M, E)$.

The space $C \ell_{\log , q}(M, E)$ is stable under the adjoint action of $C \ell^{*}(M, E)$ for if $A=\frac{a}{q} \log _{\theta} Q+A^{0}$ with $A^{0} \in C \ell^{0}(M, E)$ then for any $P \in C \ell^{*}(M, E)$ we have

$$
\begin{aligned}
P^{-1} A P & =\frac{a}{q} P^{-1} \log _{\theta} Q P+P^{-1} A_{0} P \\
& =\frac{a}{q} \log _{\theta}\left(P^{-1} Q P\right)+P^{-1} A_{0} P
\end{aligned}
$$

which lies in $C \ell_{\log , q}(M, E)$ since $P^{-1} A_{0} P$ lies in $C \ell^{0}(M, E)$ and $P^{-1} Q P$ is an operator in $C \ell^{*}(M, E)$ with same spectral cut and same order as $Q$.

This uses the fact that $P^{-1} \log _{\theta} Q P=\log _{\theta}\left(P^{-1} Q P\right)$ which we now check. We have $\log _{\theta}\left(P^{-1} Q P\right)=\left.\frac{d}{d z}\right|_{z=0}\left(P^{-1} Q P\right)_{\theta}^{z}$ with

$$
\begin{aligned}
\left(P^{-1} Q P\right)_{\theta}^{z} & =\frac{i}{2 \pi} \int_{\Gamma_{\theta}} \lambda^{z}\left(P^{-1} \log _{\theta} Q P-\lambda\right)^{-1} d \lambda \\
& =P^{-1}\left(\frac{i}{2 \pi} \int_{\Gamma_{\theta}} \lambda^{z}(Q-\lambda)^{-1} d \lambda\right) P \\
& =P^{-1} Q_{\theta}^{z} P
\end{aligned}
$$

from which it follows that $\log _{\theta}\left(P^{-1} Q P\right)=P^{-1} \log _{\theta} Q P$.

The existence of a decomposition $A=\frac{a}{q} \log _{\theta} Q+A^{0}, \quad A^{0} \in C \ell^{0}(M, E)$ follows from the fact that $A-\frac{a}{q} \log _{\theta} Q \in C \ell^{0}(M, E)$ since $\sigma_{A-\frac{a}{q}} \log _{\theta} Q=\sigma_{A}-$ $\frac{a}{q} \sigma_{\log _{\theta} Q}=a \log |\xi|+\sigma_{A}^{0}-\frac{a}{q}\left(q|\xi|+\sigma_{\log _{\theta} Q}^{0}\right)$ lies in $C S^{0}(U, W)$. The uniqueness of the decomposition for given order $a$ and given $Q$ follows in a straight forward way.

A computation on the level of symbols shows that for any $A \in C \ell^{0}(M, E)$ the operator bracket $\left[A, \log _{\theta} Q\right]$ lies in $C \ell^{0}(M, E)$ so that the bracket has a natural extension to $C \ell_{\log }(M, E)$ given by

$$
\left[\alpha \log _{\theta} Q+\widetilde{A^{0}, \beta} \log _{\theta} Q+B^{0}\right]:=\alpha\left[\log _{\theta} Q, B^{0}\right]-\beta\left[\log _{\theta} Q, A^{0}\right]+\left[A^{0}, B^{0}\right]
$$

which makes $C \ell_{\log }(M, E)$ a Fréchet Lie algebra.

\subsection{Traces on $C \ell^{0}(M, E)$ extended to logarithmic operators}

An extension of the Wodzicki residue to $C \ell_{\log }(M, E)$ was built by Okikiolu [O] and then used in [Sc] to extend Wodzicki's mutliplicative residue to a determinant on operators of any order in $C \ell^{*}(M, E)$ with spectral cut. One defines the Wodzicki residue of a logarithmic symbol $\sigma=\sigma^{0}+q \log |\xi| \in C S(U, W)$ by

$$
\begin{aligned}
\widetilde{\operatorname{res}_{x}}(\sigma) & =\int_{|\xi|=1}\left(\operatorname{tr}_{x}(\sigma)\right)_{-n} d_{S} \xi \\
& =\int_{|\xi|=1}\left(\operatorname{tr}_{x}\left(\sigma^{0}\right)\right)_{-n} d_{S} \xi .
\end{aligned}
$$

for any point $x \in U$. 
Remark 12 This differs from the higher residue of $\sigma$ seen as a logpolyhomogeneous symbols [Le] which vanishes on $\sigma \in C S_{\log }(U, W)$ :

$$
\begin{aligned}
\operatorname{res}_{x, 1}(\sigma) & =\int_{|\xi|=1}\left(\operatorname{tr}_{x}\left(\sigma^{1}\right)\right)_{-n} d_{S} \xi \\
& =0
\end{aligned}
$$

For any logarithmic operator $A \in C \ell_{\log }(M, E)$ with symbol $\sigma_{A} \in C S(U, W)$ in a local trivialisation $E_{\left.\right|_{U}} \simeq U \times W$, the local form $\operatorname{res}_{x}\left(\sigma_{A}\right) d x$ is in fact globally defined.

Lemma 9 [?] The Wodzicki residue on $C \ell^{0}(M, E)$ extends to a continuous linear form $\widetilde{\text { res }}$ on $C \ell_{\log }(M, E)$ defined as follows:

$$
\widetilde{\operatorname{res}}(A):=\frac{1}{(2 \pi)^{n}} \int_{M} d x \widetilde{\operatorname{res}_{x}}\left(\sigma_{A}\right) d x
$$

which is invariant under the adjoint action of $C \ell^{*}(M, E)$ on $C \ell_{\log }(M, E)$.

Proof: The continuity follows from the fact that $\widetilde{\text { res }}$ only depends on a finite number of homogeneous components in the symbol. Recall that given an operator $Q \in C \ell^{*}(M, E)$ with spectral cut $\theta$ and positive order $q$ and any $A=$ $A^{0}+\frac{a}{q} \log _{\theta} Q \in C \ell_{\log , a}(M, E)$ then $P^{-1} A P=P^{-1} A^{0} P+\frac{a}{q} \log _{\theta}\left(P^{-1} Q P\right)$. Since the restriction of the Wodzicki residue to zero order operators was proved to be Ad-invariant, we have $\widetilde{\operatorname{res}}\left(P^{-1} A^{0} P\right)=\operatorname{res}\left(P^{-1} A^{0} P\right)=\operatorname{res}(A)$. In order to prove the Ad-invariance of $\widetilde{\text { res }}$ on the class $C \ell_{\log }(M, E)$ of logarithmic operators, it is therefore sufficient to check that

$$
\widetilde{\operatorname{res}}\left(\log _{\theta}\left(P^{-1} Q P\right)\right)=\widetilde{\operatorname{res}}\left(\log _{\theta}(Q)\right)
$$

This can be shown using an alternative description of the extended residue $\widetilde{\text { res }}$ namely $[\mathrm{Sc}]$ :

$$
\begin{aligned}
\widetilde{\operatorname{res}}\left(\log _{\theta}\left(P^{-1} Q P\right)\right) & =\zeta_{P^{-1} Q P, \theta}(0) \\
& :=\lim _{z \rightarrow 0} \operatorname{TR}\left(\left(P^{-1} Q P\right)_{\theta}^{z}\right) \\
& =\lim _{z \rightarrow 0} \operatorname{TR}\left(Q_{\theta}^{z}\right) \\
& =\zeta_{Q}(0)
\end{aligned}
$$

where we have used the Ad-invariant of the canonical trace TR.

Similarly, leading symbol traces extend to $C \ell_{\log }(M, E)$.

Lemma 10 Leading symbol traces $\operatorname{tr}_{0}^{\tau}$ extend to continuous linear forms $\widetilde{\operatorname{tr}_{0}^{\tau}}$ on $C \ell_{\log }(M, E)$ defined as follows:

$$
\widetilde{\operatorname{tr}_{0}^{\tau}}(A):=\tau\left(\left(\operatorname{tr}_{x}\left(\sigma_{A}\right)\right)_{0}\right)=\tau\left(\left(\operatorname{tr}_{x}\left(\sigma_{A}\right)\right)_{0}^{0}\right)
$$

which are invariant under the adjoint action of $C \ell^{*}(M, E)$. 
Proof: To check that this expression makes sense for any logarithmic operator $A$, it is sufficient to check that it makes sense on logarithms of operators in $C \ell^{*}(M, E)$ with spectral cut. An operator $Q \in C \ell^{*}(M, E)$ with spectral cut $\theta$. has a well defined power $Q_{\theta}^{z}$ which we simply denote by $Q^{z}$.

Since the homogeneous components of the symbol of $Q^{z}$ satisfy

$$
\left(\sigma_{Q^{z}}(x, \xi)\right)_{q z-j}=|\xi|^{q z-j}\left(\sigma_{Q^{z}}\left(x, \frac{\xi}{|\xi|}\right)\right)_{q z-j}
$$

differentiating at $z=0$ yields for any non negative integer $j$

$$
\begin{aligned}
& \frac{d}{\left.d z\right|_{z=0}}\left(\sigma_{Q^{z}}(x, \xi)\right)_{q z-j} \\
= & \frac{d}{\left.d z\right|_{z=0}}\left(|\xi|^{q z-j} \sigma_{Q^{z}}\left(x, \frac{\xi}{|\xi|}\right)\right)_{q z-j} \\
= & q \log |\xi|\left(\sigma_{Q^{z}}(x, \xi)\right)_{q z-j}+\left.|\xi|^{-j} \frac{d}{d z}\right|_{z=0}\left(\sigma_{Q^{z}}\left(x, \frac{\xi}{|\xi|}\right)\right)_{q z-j}
\end{aligned}
$$

so that restricting to the unit cosphere $|\omega|=1$ we have

$$
\begin{aligned}
\left(\sigma_{\log Q}\right)_{0}(x, \omega) & =\frac{d}{\left.d z\right|_{z=0}}\left(\sigma_{Q^{z}}(x, \omega)\right)_{q z} \\
& =\left.\frac{d}{d z}\right|_{\left.\right|_{z=0}} \sigma_{Q^{z}}^{L}(x, \omega)
\end{aligned}
$$

where the upper index $L$ stands for the leading symbol. Since the leading symbol of $\sigma_{Q^{z}}$ is globally defined on $T^{*} M$ so is its restriction of $\left(\sigma_{\log Q}\right)_{0}$ to the unit cosphere $S^{*} M$ from which it follows that $\operatorname{tr}_{0}^{\tau}(Q):=\tau\left(\left(\operatorname{tr}_{x}\left(\sigma_{Q}\right)\right)_{0}\right)$ is well defined.

It also follows from there that the extended leading symbol trace is Ad-invariant, namely that for any $P \in C \ell^{*}(M, E)$, we have $\widetilde{\operatorname{tr}_{0}^{\tau}}\left(P^{-1} A P\right)=\widetilde{\operatorname{tr}_{0}^{\tau}}(A)$. To show this, it is sufficient to check that $\widetilde{\operatorname{tr}_{0}^{\tau}}\left(\log _{\theta}\left(P^{-1} \log _{\theta} Q P\right)=\widetilde{\operatorname{tr}_{0}^{\tau}}\left(\log _{\theta} Q\right)\right.$ which follows from

$$
\begin{aligned}
\widetilde{\operatorname{tr}_{0}^{\tau}}\left(\log _{\theta}\left(P^{-1} \log _{\theta} Q P\right)\right) & =\tau\left(\operatorname { t r } _ { x } \left(\sigma_{\left.\left.\log _{\theta}\left(P^{-1} \log _{\theta} Q P\right)\right)_{0}\right)}\right.\right. \\
& =\left.\frac{d}{d z}\right|_{z=0} \tau\left(\operatorname{tr}_{x}\left(\sigma_{\left(P^{-1} Q P\right)_{\theta}^{z}}^{L}\right)\right) \\
& =\left.\frac{d}{d z}\right|_{z=0} \tau\left(\operatorname{tr}_{x}\left(\sigma_{P^{-1}}^{L} \sigma_{Q_{\theta}^{z}}^{L} \sigma_{P}^{L}\right)\right) \\
& =\left.\frac{d}{d z}\right|_{z=0} \tau\left(\operatorname{tr}_{x}\left(\sigma_{Q_{\theta}^{z}}^{L}\right)\right) \\
& =\tau\left(\operatorname{tr}_{x}\left(\sigma_{\log _{\theta} Q}\right)_{0}\right) \\
& =\widetilde{\operatorname{tr}_{0}^{\tau}}\left(\log _{\theta} Q\right)
\end{aligned}
$$

where we have used the multiplicativity of the leading symbol together with the invariance of the ordinary trace on matrices under the adjoint action of the linear group. 
Proposition 7 Linear forms $\tilde{\lambda}: C \ell_{\log }(M, E) \rightarrow \mathbb{C}$ which restrict to a linear form $\lambda$ on $C \ell^{0}(M, E)$ are uniquely determined by a constant $c=\frac{\tilde{\lambda}\left(\log _{\theta} Q\right)}{q}$ for some fixed operator $Q \in C \ell^{*}(M, E)$ with spectral cut $\theta$ and positive order $q$. For $A=A^{0}+\frac{a}{q} \log _{\theta} Q \in C \ell_{\log , a}(M, E)$ we have

$$
\tilde{\lambda}(A)=\lambda\left(A^{0}\right)+a c .
$$

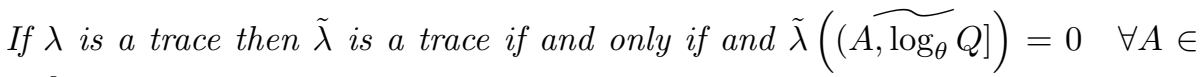
$C \ell^{0}(M, E)$.

Proof: An operator $A \in C \ell_{\log , a}(M, E)$ decomposes in a unique manner as $A=A^{0}+\frac{a}{q} \log _{\theta} Q$ so that

$$
\tilde{\lambda}(A)=\lambda\left(A^{0}\right)+a c
$$

with $c=\frac{\tilde{\lambda}\left(\log _{\theta} Q\right)}{q}$. This extension $\tilde{\lambda}$ does not depend on the choice of $Q$ made to decompose $A$. Indeed, given another operator $Q^{\prime} \in C \ell^{*}(M, E)$ with spectral cut $\theta^{\prime}$ and order $q^{\prime}>0$, the decompositions $A=A^{0}+\frac{a}{q} \log _{\theta} Q$ using $Q$ and $A=\left(A^{0}\right)^{\prime}+\frac{a}{q^{\prime}} \log _{\theta^{\prime}} Q^{\prime}$ using $Q^{\prime}$ compare as follows:

$$
\left(A^{0}\right)^{\prime}-A^{0}=a\left(\frac{\log _{\theta} Q}{q}-\frac{\log _{\theta^{\prime}} Q^{\prime}}{q^{\prime}}\right) \in C \ell^{0}(M, E) .
$$

The linearity of $\tilde{\lambda}$ implies that

$$
\begin{aligned}
\lambda\left(A^{0}\right)+\frac{a}{q} \tilde{\lambda}\left(\log _{\theta} Q\right) & =\lambda\left(A^{0}\right)+\lambda\left(\frac{a}{q} \log _{\theta} Q-\frac{a}{q^{\prime}} \log _{\theta^{\prime}} Q^{\prime}\right) \\
& +\frac{a}{q^{\prime}} \tilde{\lambda}\left(\log _{\theta^{\prime}} Q^{\prime}\right) \\
& =\lambda\left(\left(A^{0}\right)^{\prime}\right)+\frac{a}{q^{\prime}} \tilde{\lambda}\left(\log _{\theta^{\prime}} Q^{\prime}\right)
\end{aligned}
$$

where

$$
\tilde{\lambda}\left(\log _{\theta^{\prime}} Q^{\prime}\right)=\frac{q^{\prime}}{q} \lambda\left(\log _{\theta} Q\right)+\lambda\left(\log _{\theta^{\prime}} Q^{\prime}-\frac{q^{\prime}}{q} \log _{\theta^{\prime}} Q^{\prime}\right)
$$

is entirely determined by $\lambda$ and $\tilde{\lambda}\left(\log _{\theta} Q\right)$.

\subsection{Continuous paths of varying order}

Along the lines of [KV1, KV2] (see also [Le]), we define a notion of regularity of paths in $C \ell(M, E)=\bigcup_{m \in \mathbb{R}} C \ell^{m}(M, E)$ of varying order that extends the regularity of paths in $C \ell^{0}(M, E)$ given by the Fréchet topology.

Definition 4 A path $\{A(t), t \in \mathbb{R}\}$ in $C \ell(M, E)$ of classical pseudodifferential operators of order $t \mapsto \alpha(t)$ is continuous (resp. k-times differentiable, resp. of class $C^{k}$ ) provided it satisfies the following conditions 
- its order $t \mapsto \alpha(t)$ is continuous (resp. k-times differentiable, resp. of class $C^{k}$ )

- With the notations of equation (1), in each local chart $U_{i}$, and for every non negative integer $j$, the map $t \mapsto \sigma_{\alpha(t)-j}^{(i)}(A(t)) \in C^{\infty}\left(U_{i} \times \mathbb{R}^{n}\right)$ is continuous (resp. $k$-times differentiable, resp. of class $C^{k}$ )

- the map $t \mapsto K_{N}^{(i)}(A(t)) \in C^{M(N)}\left(U_{i} \times \mathbb{R}^{n}\right)$ is continuous (resp. k-times differentiable, resp. of class $C^{k}$ ) with $M(N) \rightarrow \infty$ as $N \rightarrow \infty$.

Remark 13 - Continuous paths in $C \ell^{0}(M, E)$ for the Fréchet topology are continuous as constant order paths in $C \ell(M, E)$.

- Paths of the type $A(t)=A+t B, t \in \mathbb{R}$ with $b=\operatorname{ord}(B)>a=\operatorname{ord}(A)$ are not continuous in the above sense for their order is not continuous at $t=0$.

Proposition 8 Let $t \mapsto A(t) \in C \ell(M, E)$ be a differentiable path of order $t \mapsto \alpha(t)$ then $\dot{A}(t)$ lies in $C \ell^{*, 1}(M, E)$. If $A(t) \in C \ell^{*}(M, E)$ then $A(t)^{-1} \dot{A}(t)$ lies in $C \ell_{\log , \alpha^{\prime}(t)}(M, E)$.

Proof: Let $\sigma(t)$ denote the local symbol of $A(t)$ in a local chart $U$ of $M$ above which $E$ trivialises as $E_{\left.\right|_{U}} \simeq U \times W$. Since $\sigma(t)(x, \xi)=\sum_{j=0}^{N} \psi(\xi) \sigma_{\alpha(t)-j}(t)(x, \xi)+$ $\sigma_{(N)}(t)(x, \xi)$, whereby $\sigma_{(N)}$ has kernel in $C^{K(N)}(U \times U, W)$ and $\sigma_{\alpha(t)-j} \in$ $C^{\infty}\left(T^{*} U, W\right)$ is positively homogeneous of degree $\alpha(t)-j$, differentiating w.r.t. $t$ we find:

$$
\frac{d}{d t} \sigma(t)(x, \xi)=\sum_{j=0}^{N} \psi(\xi) \frac{d}{d t} \sigma_{\alpha(t)-j}(z)(x, \xi)+\frac{d}{d t} \sigma_{(N)}(t)(x, \xi) .
$$

Moreover,

$$
\begin{aligned}
\frac{d}{d t} \sigma_{\alpha(t)-j}(t)(x, \xi) & =\frac{d}{d t}\left(|\xi|^{\alpha(t)-j} \sigma_{\alpha(z)-j}(t)\left(x, \frac{\xi}{|\xi|}\right)\right) \\
& =\alpha^{\prime}(t) \sigma_{\alpha(t)-j}(t)(x, \xi) \cdot \log |\xi| \\
& +|\xi|^{\alpha(t)-j} \frac{d}{d t}\left(\sigma_{\alpha(t)-j}(t)\left(x, \frac{\xi}{|\xi|}\right)\right)
\end{aligned}
$$


Hence

$$
\begin{aligned}
\frac{d}{d t} \sigma(t)(x, \xi) & =\sum_{j=0}^{N} \psi(\xi) \frac{d}{d t} \sigma_{\alpha(t)-j}(z)(x, \xi)+\frac{d}{d t} \sigma_{(N)}(t)(x, \xi) \\
& =\alpha^{\prime}(t)\left(\sigma(t)-\sigma_{(N)}(t)\right) \cdot \log |\xi| \\
& +\sum_{j=0}^{N} \psi(\xi)|\xi|^{\alpha(t)-j} \frac{d}{d t}\left(\sigma_{\alpha(t)-j}(t)\left(x, \frac{\xi}{|\xi|}\right)\right) \\
& +\frac{d}{d t} \sigma_{(N)}(t)(x, \xi) \\
& =\alpha^{\prime}(t)\left(\sigma(t)-\sigma_{(N)}(t)\right) \star \log |\xi| \\
& +\sum_{j=0}^{N} \psi(\xi)|\xi|^{\alpha(t)-j} \frac{d}{d t}\left(\sigma_{\alpha(t)-j}(t)\left(x, \frac{\xi}{|\xi|}\right)\right) \\
& +\frac{d}{d t} \sigma_{(N)}(t)(x, \xi)
\end{aligned}
$$

where we have replaced the ordinary product by the star product of symbols in the last equality. It follows that $\frac{d}{d t} \sigma(t) \in C S^{*, 1}(U, W)$ and hence $\dot{A}(t) \in$ $C \ell^{*, 1}(M, E)$.

Consequently, if $A(t)^{-1}$ has symbol $\tilde{\sigma}(t)$ then $A^{-1} \frac{d}{d t} A(t)$ has symbol

$$
\begin{aligned}
\tilde{\sigma}(t) \star \frac{d}{d t} \sigma(t) & =\alpha^{\prime}(t)\left(\tilde{\sigma}(t) \star \sigma(t)-\tilde{\sigma}(t) \star \sigma_{(N)}(t)\right) \star \log |\xi| \\
& +\tilde{\sigma}(t) \star \sum_{j=0}^{N} \psi(\xi)|\xi|^{\alpha(t)-j} \frac{d}{d t}\left(\sigma_{\alpha(t)-j}(t)\left(x, \frac{\xi}{|\xi|}\right)\right) \\
& +\left(\tilde{\sigma}(t) \star \frac{d}{d t} \sigma_{(N)}(t)\right)(x, \xi) \\
& =\alpha^{\prime}(t) \log |\xi| \\
& +\left(\tilde{\sigma}(t) \star \sum_{j=0}^{N} \psi(\xi)|\xi|^{\alpha(t)-j} \frac{d}{d t}\left(\sigma_{\alpha(t)-j}\right)(t)\right)\left(x, \frac{\xi}{|\xi|}\right) \\
& -\left(\tilde{\sigma}(t) \star \sigma_{(N)}(t)\right)(x, \xi) \cdot \log |\xi| \\
+ & \left(\tilde{\sigma}(t) \star \frac{d}{d t} \sigma_{(N)}(t)\right)(x, \xi) .
\end{aligned}
$$

The only two terms involving logarithms are $\alpha^{\prime}(t) \log |\xi|$ and $\left(\tilde{\sigma}(t) \star \sigma_{(N)}(t)\right)$. $\log |\xi|$. Since $N$ can be chosen arbitrarily large and since the terms not involving any logarithms are of order 0 , it follows that

$$
\tilde{\sigma}(t) \star \frac{d}{d t} \sigma(t)-\alpha^{\prime}(t) \log |\xi| \in C S^{0}(U, W)
$$

as a consequence of which $\frac{d}{d t} \sigma_{\alpha(t)-j}(t)(x, \xi)$ lies in $C S_{\log , \alpha^{\prime}(t)}(U, W)$ and $A(t)^{-1} \frac{d}{d t} A(t)$ in $C \ell_{\log , \alpha^{\prime}(t)}(M, E)$.

We define

$$
\mathcal{P} C \ell^{*}(M, E):=\left\{\gamma \in C^{1}\left([0,1], C \ell^{*}(M, E)\right), \quad \gamma(0)=1\right\} .
$$


Let $\tilde{C} \ell^{*}(M, E)$ denote the pathwise connected component of the identity in $C \ell^{*}(M, E)$.

The adjoint action of zero order invertible classical pseudodifferential operators on $C \ell^{0}(M, E)$ extends to invertible classical pseudodifferential operators of any order, namely for any $P \in C \ell^{*}(M, E)$ we set

$$
\begin{aligned}
A d_{P}: C \ell^{m}(M, E) & \rightarrow C \ell^{m}(M, E) \\
A & \mapsto A d_{P}(A)=P^{-1} A P .
\end{aligned}
$$

The subgroup $\tilde{C} \ell^{*}(M, E)$ is normal in $C \ell^{*}(M, E)$ so that the adjoint action of $C \ell^{*}(M, E)$ preserves $\tilde{C} \ell^{*}(M, E)$.

Proposition 9 The maps

$$
\begin{aligned}
F_{s}: C \ell^{*}(M, E) & \rightarrow C \ell^{*}(M, E) \\
A & \mapsto A|A|^{-s}, \quad s \in[0,1]
\end{aligned}
$$

provide a homotopy from from $C \ell^{*}(M, E)$ to $C \ell^{0, *}(M, E)$.

Proof: Given any $A \in C \ell^{*}(M, E)$, then $A^{*} A$ has spectral cut $\theta=\pi$ so that we can define

$$
|A|:=\frac{1}{2 i \pi} \int_{\Gamma_{\pi}} \mu^{\frac{1}{2}}\left(A^{*} A-\mu\right)^{-1} d \mu
$$

where $\Gamma_{\pi}$ is a contour around the (positive) spectrum of $A$. Let us set $U:=$ $A|A|^{-1}$ which lies in $C \ell^{0}(M, E)$. Then $\gamma_{A}(s):=A|A|^{-s}$ is a continuous path in $C \ell^{*}(M, E)$ of operators of order $s \cdot a$ where $a$ is the order of $A$ which links $A$ to $U \in C \ell^{0, *}(M, E)$ so that the map $F_{s}$ indeed provides a homotopy.

\subsection{A uniqueness result for mutliplicative determinants on operators of all orders}

From the previous paragraphs we know that any continuous trace $\lambda$ on $C \ell^{0}(M, E)$ can be extended to a continuous linear form $\tilde{\lambda}$ on $C \ell_{\log }(M, E)$ so that it makes sense to define

$$
\begin{aligned}
\operatorname{Det}_{\tilde{\lambda}}: \mathcal{P} C \ell^{*}(M, E) & \rightarrow \mathbb{C}^{*} \\
\gamma & \mapsto e^{\int_{0}^{1} \tilde{\lambda}\left(\dot{\gamma} \gamma^{-1}\right)}
\end{aligned}
$$

Lemma 11 For any of the extended traces $\tilde{\lambda}=\widetilde{\operatorname{res}}$ and $\tilde{\lambda}=\widetilde{\operatorname{tr}_{0}^{\tau}}$, Det $\tilde{\lambda}$ is trivial on loops in $\mathcal{P} C \ell^{*}(M, E)$.

Proof: This follows from combining Lemma 6 with Proposition 9.

As a result, similarly to the case of zero order operators, one can define an associated determinant:

$$
\begin{aligned}
\operatorname{det}_{\tilde{\lambda}}: \tilde{C} \ell^{*}(M, E) & \rightarrow \mathbb{C}^{*} \\
A & \mapsto \operatorname{Det}_{\tilde{\lambda}}\left(\gamma_{A}\right)
\end{aligned}
$$


independently of the choice of path $\gamma_{A} \in \mathcal{P} C \ell^{*}(M, E)$ such that $\gamma_{A}(1)=A$.

Theorem 6 1. If a continuous trace $\lambda$ on $C \ell^{0}(M, E)$ extends to a linear map $\tilde{\lambda}$ on $C \ell_{\log }(M, E)$ which is invariant under the adjoint action of $C \ell^{*}(M, E)$ then the corresponding determinant map $\operatorname{det}_{\lambda}$ on $\tilde{C} \ell^{*, 0}(M, E)$ extends to a multiplicative map $\operatorname{det}_{\tilde{\lambda}}: \tilde{C} \ell^{*}(M, E) \rightarrow \mathbb{C}^{*}$ which is continuous along continuous paths of $\tilde{C} \ell^{*}(M, E)$. Determinant maps $\operatorname{det}_{\tilde{\lambda}}$ and $\operatorname{det}_{\bar{\lambda}}$ corresponding to two extensions $\tilde{\lambda}$ and $\bar{\lambda}$ of $\lambda$ compare as follows. There is a constant $K$ such that

$$
\operatorname{det}_{\tilde{\lambda}}(A)=K^{a} \operatorname{det}_{\bar{\lambda}}(A) \quad \forall A \in \tilde{C} \ell^{*, a}(M, E) .
$$

2. Conversely, let $\Lambda: \tilde{C} \ell^{*}(M, E) \rightarrow \mathbb{C}^{*}$ be a multiplicative map, the restriction $\Lambda^{0}:=\Lambda_{\left.\right|_{C \ell^{0, *}(M, E)}}$ of which is of class $C^{1}$. Then

$$
\lambda:=D_{1} \Lambda^{0}
$$

is a continuous trace on $C \ell^{0}(M, E)$ and

$$
\Lambda^{0}=\operatorname{det}_{\lambda}
$$

is the associated determinant on zero order operators.

If moreover $\Lambda$ is continuous along continuous paths in $\tilde{C} \ell^{*}(M, E)$, then it can be expressed in terms of any extended determinant $\operatorname{det}_{\tilde{\lambda}}$ defined by some extension $\tilde{\lambda}$ of $\lambda$ as in 1. as follows:

$$
\Lambda(A)=K^{a} \operatorname{det}_{\tilde{\lambda}}(A)
$$

on operators $A \in \tilde{C} \ell^{*}(M, E)$ of order a where $K$ is some constant depending on $\Lambda$ and $\tilde{\lambda}$.

Remark 14 Note that for $A$ of order 0 , the last formula yields back $\Lambda^{0}(A)=$ $\operatorname{det}_{\tilde{\lambda}}(A)=\operatorname{det}_{\lambda}(A)$.

\section{Proof:}

1. We proceed as in the proof of Proposition 2 starting with the proof of the multiplicativity property of $\operatorname{det}_{\lambda}$. Let $\gamma_{1}, \gamma_{2} \in \mathcal{P} C \ell^{*}(M, E)$ be differentiable paths of orders $\alpha_{1}(t), \alpha_{2}(t)$ respectively. Then

$$
\begin{aligned}
\tilde{\lambda}\left(\left(\gamma_{1} \gamma_{2}\right)^{-1} \frac{d}{d t}\left(\gamma_{1} \gamma_{2}\right)\right) & =\tilde{\lambda}\left(A d_{\gamma_{2}}\left(\gamma_{1}^{-1} \dot{\gamma}_{1}\right)\right)+\tilde{\lambda}\left(\gamma_{2}^{-1} \dot{\gamma}_{2}\right) \\
& =\tilde{\lambda}\left(\dot{\gamma}_{1} \gamma_{1}^{-1}\right)+\tilde{\lambda}\left(\dot{\gamma}_{2} \gamma_{2}^{-1}\right),
\end{aligned}
$$

where we have used the Ad-invariance of $\tilde{\lambda}$ under the adjoint action $A d_{\gamma_{1}}$ in the second identity. Hence

$$
\operatorname{Det}_{\tilde{\lambda}}\left(\gamma_{1} \gamma_{2}\right)=\operatorname{Det}_{\tilde{\lambda}}\left(\gamma_{1}\right) \operatorname{Det}_{\tilde{\lambda}}\left(\gamma_{2}\right) \text {. }
$$

Since $\tilde{\lambda}$ restricts to $\lambda$ on zero order pseudodifferential operators, $\operatorname{det}_{\tilde{\lambda}}$ restricts to $\operatorname{det}_{\tilde{\lambda}}^{0}=\operatorname{det}_{\lambda}$ on zero order pseudodifferential operators so that 
the proof of the differentiability at 1 of the restriction of $\operatorname{det}_{\tilde{\lambda}}^{0}$ goes as in the proof of Proposition 2 which yields $D_{1} \operatorname{det}_{\tilde{\lambda}}^{0}=\lambda$.

The continuity of $\tilde{\lambda}$ on $C \ell_{\log }(M, E)$ then implies that of $\operatorname{det}_{\tilde{\lambda}}$ along continuous paths in $C \ell^{*}(M, E)$.

Two extensions $\tilde{\lambda}$ and $\bar{\lambda}$ of $\lambda$ differ on $C \ell_{\log , a}(M, E)$ by $\tilde{\lambda}-\bar{\lambda}=a c$ with $c=\frac{1}{q}\left(\tilde{\lambda}\left(\log _{\theta} Q\right)-\bar{\lambda}\left(\log _{\theta} Q\right)\right)$ for some $Q \in C \ell^{*}(M, E)$ with positive order $q$ and spectral cut $\theta$. It follows that the corresponding determinants differ for an operator $A$ of order $a$ by $\operatorname{det}_{\tilde{\lambda}}(A)=K^{a} \operatorname{det}_{\bar{\lambda}}(A)$ where we have set $K=e^{c}=e^{\frac{1}{q}\left(\tilde{\lambda}\left(\log _{\theta} Q\right)-\bar{\lambda}\left(\log _{\theta} Q\right)\right)}$.

2. Conversely, starting from a multiplicative map $\Lambda: \tilde{C} \ell^{*}(M, E) \rightarrow \mathbb{C}^{*}$, the restriction $\Lambda^{0}:=\Lambda_{\left.\right|_{C \ell^{0}, *(M, E)}}$ of which is differentiable at 1 , we set $\lambda:=D_{1} \Lambda^{0}$. As in the case of zero order operators, for any $A \in \tilde{C} \ell^{*}(M, E)$ and any $U \in C \ell^{0}(M, E)$ we have

$$
\begin{aligned}
\operatorname{Ad}_{A}^{*} \lambda(U) & =\left.\frac{d}{d t}\right|_{t=0} \operatorname{Ad}_{A}^{*} \Lambda^{0}(\operatorname{Exp}(t U)) \\
& =\left.\frac{d}{d t}\right|_{t=0} \Lambda^{0}\left(A^{-1} \operatorname{Exp}(t U) A\right) \\
& =\left.\frac{d}{d t}\right|_{t=0}\left(\Lambda(A)^{-1} \Lambda(\operatorname{Exp}(t U)) \Lambda(A)\right) \\
& =\left.\frac{d}{d t}\right|_{t=0} \Lambda(\operatorname{Exp}(t U)) \\
& =\lambda(U) .
\end{aligned}
$$

It follows that $\lambda$ is a continuous linear map on $C \ell^{0}(M, E)$ invariant under the adjoint action of $C \ell^{*}(M, E)$. Moreover, by Theorem 5 , we have $\Lambda^{0}=$ $\operatorname{det}_{\lambda}$.

The multiplicativity of $\Lambda$ then yields for any $A \in \tilde{C} \ell^{*}(M, E)$ of order $a$, any $Q \in C \ell^{*}(M, E)$ with spectral cut $\theta$ and of order $q \neq 0$ and any linear extension $\tilde{\lambda}$ of $\lambda$ :

$$
\begin{aligned}
\Lambda(A) & =\Lambda\left(A Q_{\theta}^{-\frac{a}{q}}\right) \Lambda\left(Q_{\theta}^{\frac{a}{q}}\right) \\
& =\Lambda^{0}\left(A Q_{\theta}^{-\frac{a}{q}}\right) \cdot \Lambda\left(Q_{\theta}^{\frac{a}{q}}\right) \\
& =\operatorname{det}_{\lambda}\left(A Q_{\theta}^{-\frac{a}{q}}\right) \Lambda\left(Q_{\theta}^{\frac{a}{q}}\right) \\
& =\operatorname{det}_{\tilde{\lambda}}\left(A Q_{\theta}^{-\frac{a}{q}}\right) \Lambda\left(Q_{\theta}^{\frac{a}{q}}\right) \\
& =\frac{\Lambda\left(Q_{\theta}^{\frac{a}{q}}\right)}{\operatorname{det}_{\tilde{\lambda}}\left(Q_{\theta}^{\frac{a}{q}}\right)} \cdot \operatorname{det}_{\tilde{\lambda}}(A)
\end{aligned}
$$

using the multiplicativity of $\operatorname{det}_{\tilde{\lambda}}$. For fixed extension $\tilde{\lambda}$ and fixed operator $Q$, the constant

$$
C(a):=\frac{\Lambda\left(Q_{\theta}^{\frac{a}{q}}\right)}{\operatorname{det}_{\tilde{\lambda}}\left(Q_{\theta}^{\frac{a}{q}}\right)}
$$


only depends on $A$ via its order $a$ and we have $\Lambda(A)=C(a) \operatorname{det}_{\tilde{\lambda}}(A)$. Given two operators $A, B \in \tilde{C} \ell^{*}(M, E)$ of order $a, b$ respectively, the multiplicativity of $\Lambda$ and $\operatorname{det}_{\tilde{\lambda}}$ yields:

$$
\begin{aligned}
C(a+b) \operatorname{det}_{\tilde{\lambda}}(A B) & =\Lambda(A B) \\
& =\Lambda(A) \Lambda(B) \\
& =C(a) C(b) \operatorname{det}_{\tilde{\lambda}}(A) \operatorname{det}_{\tilde{\lambda}}(B) \\
& =C(a) C(b) \operatorname{det}_{\tilde{\lambda}}(A B)
\end{aligned}
$$

so that $C(a+b)=C(a) C(b)$. It follows that $\log C$ is an additive morphism. $C$ being continuous in $a$ as a result of the continuity of $\Lambda$ and $\tilde{\lambda}$, it follows that $C(a)=K^{a}$ for some complex number $K(\Lambda, \tilde{\lambda})$ depending on $\Lambda$ and $\tilde{\lambda}$.

Since $\widetilde{\text { res }}$ and $\widetilde{t r_{0}^{\tau}}$ are Ad-invariant continuous extensions of the Wodzciki residue and the leading symbol trace respectively, as a consequence of the first part of the above theorem we can define two types of multiplicative determinants:

Definition 5 For $A \in \tilde{C} \ell^{*}(M, E)$ set

$$
\operatorname{det}_{r e s}:=\operatorname{det}_{\text {rẽs }} ; \quad \operatorname{det}_{0}^{\tau}:=\operatorname{det}_{\widetilde{\text { rr }_{0}^{\tau}}} .
$$

The following uniqueness result on multiplicative determinants is a consequence of the uniqueness result on continuous traces on $C \ell^{0}(M, E)$ (see Theorem 4) which arise as linear combinations of the Wodzicki residue and the leading symbol trace:

Theorem 7 Maps $\Lambda: \tilde{C} \ell^{*}(M, E) \rightarrow \mathbb{C}^{*}$ satisfying the following requirements

- $\Lambda$ is multiplicative,

- the restriction $\Lambda_{0}$ to $\tilde{C} \ell^{0, *}(M, E)$ is of class $C^{1}$,

- $\Lambda$ is continuous along continuous paths in $\tilde{C} \ell^{*}(M, E)$

are of the form

$$
\Lambda(A)=K^{a} \cdot \operatorname{det}_{\tilde{\lambda}}(A)
$$

on operators $A \in \tilde{C} \ell^{*}(M, E)$ of order a where $\tilde{\lambda}$ is a linear combination of $\widetilde{\text { res }}$ and $\widetilde{\mathrm{tr}_{0}^{\tau}}$.

We now specialize to admissible operators. Let $C \ell_{a d m}^{*}(M, E)$ denote the class of operators in $C \ell^{*}(M, E)$ that admit a spectral cut, called admissible operators. Note that $C \ell_{a d m}^{*}(M, E) \subset \tilde{C} \ell^{*}(M, E)$ since there is a path $\gamma_{A}(t)=A_{\theta}^{t}$ in $C \ell^{*, a d m}(M, E)$ linking $A$ to the identity where $\theta$ is a spectral cut for $A$. We set as usual:

$$
\log _{\theta} A:=\left.\frac{d}{d t}\right|_{t=0} A_{\theta}^{t} .
$$


Corollary 1

- For any $A \in C \ell_{a d m}^{*}(M, E)$ with spectral cut $\theta$ we have:

$$
\operatorname{det}_{r e s}(A)=e^{\widetilde{\mathrm{res}}\left(\log _{\theta} A\right)} ; \quad \operatorname{det}_{0}^{\tau}(A)=e^{\widetilde{\operatorname{tr}}_{0}^{\tau}\left(\log _{\theta} A\right)}
$$

- Maps $\Lambda: C \ell_{\text {adm }}^{*}(M, E) \rightarrow \mathbb{C}^{*}$ satisfying the following requirements

$-\Lambda$ is multiplicative,

- the restriction $\Lambda^{0}$ to $C \ell^{0, *}(M, E)$ is of class $C^{1}$,

$-\Lambda$ is continuous along continuous paths in $C \ell_{a d m}^{*}(M, E)$

are of the form

$$
\Lambda(A)=K^{a} \cdot e^{\tilde{\lambda}\left(\log _{\theta}(A)\right)}
$$

on operators $A \in \tilde{C} \ell^{*}(M, E)$ of order a where $\tilde{\lambda}$ is a linear combination of $\widetilde{r e s}$ and $\widetilde{\operatorname{tr}_{0}^{\tau}}$.

Proof: Let $A \in C \ell_{a d m}^{*}(M, E)$ with spectral cut $\theta$. Since $\tilde{\lambda}$ is continuous along continuous paths we have

$$
\begin{aligned}
d \tilde{\lambda}\left(\log _{\theta}(A)\right) & =\left.\frac{d}{d z}\right|_{z=0} d \tilde{\lambda}\left(A_{\theta}^{z}\right) \\
& =\left.\frac{d}{d z}\right|_{z=0} d\left(\tilde{\lambda}\left(\int_{\Gamma_{\theta}} \mu_{\theta}^{z}(A-\mu)^{-1} d \mu\right)\right) \\
& =\left.\frac{d}{d s}\right|_{z=0} \tilde{\lambda}\left(\int_{\Gamma_{\theta}} \mu_{\theta}^{z} d(A-\mu)^{-1} d \mu\right) \\
& =-\frac{d}{d z} \int_{\left.\right|_{z=0}} \mu_{\Gamma_{\theta}}^{z} \tilde{\lambda}\left((A-\mu)^{-1} d A(A-\mu)^{-1} d \mu\right) \\
& =-\frac{d}{d z} \int_{z=0} \int_{\Gamma_{\theta}} \mu_{\theta}^{z} \tilde{\lambda}\left(d A(A-\mu)^{-2} d \mu\right) \\
& =\left.\frac{d}{d z}\right|_{z=0}\left(z \tilde{\lambda}\left(\int_{\Gamma_{\theta}} \mu_{\theta}^{z-1} d A(A-\mu)^{-1} d \mu\right)\right) \\
& =\tilde{\lambda}\left(\int_{\Gamma_{\theta}} \mu^{-1} d A(A-\mu)^{-1} d \mu\right) \\
& =\tilde{\lambda}\left(A^{-1} d A\right) .
\end{aligned}
$$

The path $\gamma_{A}(t):=A_{\theta}^{t}$ lies in $\mathcal{P} C \ell^{*}(M, E)$ and $\gamma_{A}(1)=A$. Integrating the differential equation $d \tilde{\lambda}\left(\log _{\theta}(A)\right)=\tilde{\lambda}\left(A^{-1} d A\right)$ obtained above along the path $\gamma_{A}$ yields $\tilde{\lambda}\left(\log _{\theta}(A)\right)=\int_{0}^{1} \tilde{\lambda}\left(\gamma_{A}(t)^{-1} \frac{d}{d t} \gamma_{A}(t)\right)$. Now the right hand side depends on the choice of path only via its homotopy class. On the other hand, the fundamental group is generated by $\left[t \mapsto e^{2 i \pi t P}\right]$ where the $P$ are pseudifferential projectors defined above, for which res vanishes and $\operatorname{tr}_{0}^{\tau}$ lies in $2 i \pi \mathbb{Z}$. Hence $\widetilde{\operatorname{det}_{\text {res }}}\left(\log _{\theta} A\right)$ is well defined and $\widetilde{\operatorname{tr}_{0}^{\tau}}\left(\log _{\theta} A\right)$ is defined modulo $2 i \pi$. Moreover we have

$$
\operatorname{det}_{r e s}(A)=e^{\widetilde{\mathrm{res}}\left(\log _{\theta} A\right)} ; \quad \operatorname{det}_{0}^{\tau}(A)=e^{\widetilde{\mathrm{tr}_{0}^{\tau}}\left(\log _{\theta} A\right)} .
$$

With these formulae at hand, the continuity of the maps $A \mapsto \operatorname{det}_{\text {res }}\left(A_{\theta}^{t}\right)$ and $t \mapsto \operatorname{det}_{0}^{\tau}\left(A_{\theta}^{t}\right)$ follows from the continuity of the extended Wodzicki residue and 
leading symbol traces.

When $\lambda=$ res this yields back the residue determinant on admissible invertible positive order elliptic operators introduced by Scott [Sc], which arises here as an extension of the "exotic determinant" det $_{\text {res }}$ introduced by Wodzicki $[\mathrm{W}],[\mathrm{K}]$. When $\lambda=\operatorname{tr}_{0}^{\tau}$, this yields a new class of multiplicative determinants on admissible invertible positive order elliptic operators.

Let us illustrate these determinants with some examples. We recall from [Sc] that the logarithm of the residue determinant of an invertible elliptic admissible differential operator $A$ of positive order coincides with (minus) the value at $z=0$ of its $\zeta$-function $\zeta_{A}(z)$ so that

$$
\operatorname{det}_{\mathrm{res}}(A)=e^{-\zeta_{A}(0)}
$$

The residue determinant naturally induces a super residue determinant in the $\mathbb{Z}_{2}$-graded case. For a $\mathbb{Z}_{2}$-graded vector bundle $E=E^{+} \oplus E^{-}$and given an even invertible elliptic admissible differential operator $A=A^{+} \oplus A^{-}$acting on sections of $E$, we define

$$
\operatorname{sdet}_{\mathrm{res}}(A):=\frac{\operatorname{det}_{\mathrm{res}}\left(A^{+}\right)}{\operatorname{det}_{\mathrm{res}}\left(A^{-}\right)} .
$$

When $E$ is a spinor bundle over an even dimensional manifold $M$ and $A:=\Delta+1$ with $\Delta^{+} \oplus \Delta^{-}$the square of the Dirac operator, then $(21)$ yields $\operatorname{sdet}_{\text {res }}(A)=$ $e^{\zeta_{\Delta^{-+1}}(0)-\zeta_{\Delta^{++1}}(0)}=e^{- \text {ind } D^{+}}$where $D^{+}$is the chiral Dirac operator acting from sections of $E^{+}$to sections of $E^{-}$. The (super)-residue determinant therefore picks up the index which from the physics point of view typically carries the chiral anomaly.

More generally, we have the following result.

Proposition 10 Let $A, B$ be two admissible elliptic invertible operators with same order acting on smooth sections of some vector bundle $E$ over $M$. If $A$ and $B$ have scalar leading symbols then

$$
\frac{\operatorname{det}_{r e s}(A)}{\operatorname{det}_{r e s}(B)}=e^{\sum_{k=1}^{\infty}(-1)^{k+1} \frac{\operatorname{res}\left(\left(B^{-1}(A-B)\right)^{k}\right)}{k}}
$$

and

$$
\frac{\operatorname{det}_{0}^{\tau}(A)}{\operatorname{det}_{0}^{\tau}(B)}=1
$$

Proof: Since $A=B\left(1+B^{-1}(A-B)\right)$, the multiplicativity of the determinants yields

$$
\frac{\operatorname{det}_{r e s}(A)}{\operatorname{det}_{r e s}(B)}=\operatorname{det}_{r e s}\left(1+B^{-1}(A-B)\right)
$$

and similarly,

$$
\frac{\operatorname{det}_{0}^{\tau}(A)}{\operatorname{det}_{0}^{\tau}(B)}=\operatorname{det}_{0}^{\tau}\left(1+B^{-1}(A-B)\right) .
$$

Since $B$ has positive order equal to the order of $A, B^{-1}(A-B)$ has negative order so that applying Lemma 3.2 to $B^{-1}(A-B)$ yields the result.

As pointed out in the introduction, this shows in particular that the leading symbol determinant is too coarse to distinguish between different generalized Laplacians, so that it sees little of the underlying geometry. 


\section{References}

[Bo] J.B. Bost, Principe d'Oka, K-théorie et systèmes dynamiques non commutatifs, Invent. Math. 101 no. 2, 261-333 (1990)

[B] J.L. Brylinski, A differential complex for Poisson manifolds, Journ. Diff. Geom. 28 93-114 (1988)

[BG] J.L. Brylinski, E. Getzler, The homology of algebras of pseudodifferential symbols and non commutative residues, K-theory 1 385-403 (1987)

[BW] B. Booss-Bavnbek, K. Wojciechowski, Elliptic boundary problems for Dirac operators, Birkhäuser (1993)

[CE] H. Cartan, S. Eilenberg, Homological algebra, Princeton University Press 1956

[Co] A. Connes, Noncommutative Geometry, Academic Press 1994

[dLHS] P. de La Harpe, G. Skandalis, Déterminant associé à une trace sur une algèbre de Banach, Ann. Inst. Fourier, Grenoble, 34, 1 241-260 (1984)

[Ha] S. Hawkings, Zeta function regularization of path integrals on curved spacetime, Comm.Math.Phys. 13355 (1977)

[H] L. Hörmander, The analysis of linear partial differential operators. III. Pseudodifferential operators, Grundlehren der Mathematischen Wissenschaften, 274 Springer Verlag 1994

[Ka] M. Karoubi, K-theory (An introduction), Springer Verlag (1978)

[K] Ch. Kassel, Le résidu non commutatif (d'après M. Wodzicki), Séminaire Bourbaki, Astérisque 177-178 (1989) 199-229

[KM] A. Kriegel, P. W. Michor, The convenient setting of global analysis, A.M.S. Mathematical Surveys and Monographs 53 (1997)

[KV1] M. Kontsevich, S. Vishik, Geometry of determinants of elliptic operators, Func. Anal. on the Eve of the XXI century, Vol I, Progress in Mathematics 131(1994) 173-197

[KV2] M. Kontsevich, S. Vishik, Determinants of elliptic pseudodifferential operators (unpublished) arXiv-hep-th/9404046 (1994)

[Le] M.Lesch, On the noncommutative residue for pseudodifferential operators with log-polyhomogeneous symbols, Annals of Global Anal. and Geom. 17 151-187 (1999)

[Lo] J.L. Loday, Cyclic Homology, Grundlehren der mathematischen Wissenschaften, vol. 301.

[M] S. MacLane, Homology, Springer Verlag 1963

[MN] R. Melrose, V. Nistor, Homology of pseudo-differential operators I. Manifolds with boundary, funct-an/9606005, june 1999 
[O] K. Okikiolu, The multiplicative anomaly for determinants of elliptic operators, Duke Math. Journ. 79 723-750 (1995)

[Po] R. Ponge, Cayley-hamiltonian decomposition and spectral asymmetry, IHES/M/04/01 (2004); Spectral asymmetry, zeta functions and the noncommutative residue, IHES/M/04/02 (2004)

[PR1] S. Paycha, S. Rosenberg, Traces and characteristic classes in loop groups, in Infinite dimensional Groups and Manifolds, Ed. T. Wurzbacher, I.R.M.A. Lectures in Mathematical and Theoretical Physics 5, de Gruyter 185-212 (2004)

[PR2] S. Paycha, S. Rosenberg, From conformal operators to conformal anomalies via regularized traces, to appear in "Spectral and Geometric Analysis on Manifolds"-Papers in Honour of K. P. Wojciechowski", World Scientific

[RS] D.B. Ray, I.M. Singer, R-torsion and the Laplacian on Riemannian manifolds, Adv. Math. t7 145-210 (1971)

[Sc] S. Scott, The residue determinant,Commun. Part. Diff. Eqn.s, to appear, arXiv: math.AP/0406268

[Se] R.T. Seeley, Complex powers of an elliptic operator, Singular integrals, Proc. Symp. Pure Math., Chicago, Amer. Math. Soc., Providence 288-307 (1966)

[WO] N.E. Wegge-Olsen, $K$-theory and $C^{*}$-algebras, Oxford science publications (1994).

[We] C.A. Weibel, An introduction to homological algebra, Cambridge. Univ. Press (1994) 104, 19-63 (1980)

[W] M. Wodzicki, Non commutative residue, Chapter I. Fundamentals, KTheory, Arithmetic and Geometry, Lecture Notes in Math.320-399 1289 Springer Verlag 1987

[W84] M. Wodzicki, Local invariants of spectral asymmetry, Invent. Math. 75 (1984) 143-177

[W87] M. Wodzicki, Cyclic homology of differential operators, Duke Math. Journ. 54 (2), 641-647 (1987)

[W88] M. Wodzicki, Cyclic homology of pseudodifferential operators and noncommutative Euler class C.R.A.S. 306 321-325 (1998)

Jean-Marie Lescure, UMR-6620 université Blaise Pascal, COMPlexe scientifique des CÉZeaux, 63170 Aubière, France

lescure@math.univ-bpclermont.fr

Sylvie Paycha, UMR-6620 université Blaise Pascal, Complexe SCIentifique des Cézeaux, 63170 Aubière, France

paycha@math.univ-bpclermont.fr 